\title{
Homological Algebra and Divergent Series ${ }^{\star}$
}

\author{
Vassily GORBOUNOV ${ }^{\dagger}$ and Vadim SCHECHTMAN ${ }^{\ddagger}$ \\ $\dagger$ Department of Mathematical Sciences, King's College, University of Aberdeen, \\ Aberdeen, AB24 3UE, UK \\ E-mail:vgorb@maths.abdn.ac.uk \\ $\ddagger$ Laboratoire de Mathématiques Emile Picard, Université Paul Sabatier, Toulouse, France \\ E-mail: schechtman@math.ups-tlse.fr
}

Received October 01, 2008, in final form March 04, 2009; Published online March 24, 2009

doi:10.3842/SIGMA.2009.034

\begin{abstract}
We study some features of infinite resolutions of Koszul algebras motivated by the developments in the string theory initiated by Berkovits.
\end{abstract}

Key words: Koszul resolution; Koszul duality, divergent series

2000 Mathematics Subject Classification: 13D02; 14N99

To Friedrich Hirzebruch on his 80-th anniversary, with admiration

\section{Introduction}

1.1. This article consists of two parts. The first part is a simple exercise on Mellin transform. The second one is a review on some numerical aspects of Koszul duality. An object which lies behind the two parts is a Tate resolution of a commutative ring over a field of characteristic zero.

Let us give some more details on the contents. In the first part we develop the elegant ideas, due to physicists [5], which allow to define the numerical invariants of projective varieties, doing a regularization of some divergent series connected with their homogeneous rings.

Let $R_{0}=k\left[x_{0}, \ldots, x_{N}\right]$ be a polynomial algebra over a field $k, f_{1}, \ldots, f_{p} \in R_{0}$ homogeneous elements of degrees $d_{i}=\operatorname{deg} f_{i}>0$ which generate the ideal $I=\left(f_{1}, \ldots, f_{p}\right)$. Consider the quotient algebra $A=R_{0} / I$; it is graded

$$
A=\bigoplus_{j=0}^{\infty} A^{j} .
$$

Geometrically the projection $R_{0} \longrightarrow A$ corresponds to a closed embedding $i: X:=\operatorname{Proj} A \hookrightarrow$ $\mathbb{P}^{N}:=$ Proj $R_{0}$; the projective variety $X$ is defined in $\mathbb{P}^{N}$ by the equations $f_{1}=0, \ldots, f_{p}=0$. The algebra $A$ is called the homogeneous ring of $X$ (it depends on the embedding into the projective space).

Let us call a semi-free resolution of $A$ the following data:

( $i$ ) An associative unital bi-graded $k$-algebra $R=\underset{i, j=0}{\infty} R_{i}^{j}\left(\right.$ so $\left.R_{i}^{j} \cdot R_{l}^{m} \subset R_{i+l}^{j+m}\right)$; the indexes $i$ and $j$ will be called the homological degree and the polynomial degree respectively. We set $R_{i}:=\oplus_{j} R_{i}^{j}, R^{j}:=\oplus_{i} R_{i}^{j}$. We introduce a structure of a superalgebra on $R$ by defining the parity to be equal to the parity of the homological degree.

The multiplication has to be super-commutative, i.e. for $x \in R_{i}, y \in R_{l}$ we have $x y=$ $(-1)^{i l} y x$.

\footnotetext{
${ }^{\star}$ This paper is a contribution to the Special Issue on Kac-Moody Algebras and Applications. The full collection is available at http://www.emis.de/journals/SIGMA/Kac-Moody_algebras.html
} 
$R$ should be equipped with a differential $d: R \longrightarrow R, d^{2}=0$, such that $d\left(R_{i}^{j}\right) \subset R_{i-1}^{j}$ and for $x \in R_{i}, y \in R$,

$$
d(x y)=d x \cdot y+(-1)^{i} x \cdot d y .
$$

(ii) $R_{0}$ should be equipped with a morphism of algebras $\epsilon: R_{0} \longrightarrow A$ such that $\epsilon\left(R_{0}^{j}\right) \subset A^{j}$.

(iii) For each $j \geq 0, R^{j}$ should be a resolution of $A^{j}$, i.e. the complexes

$$
\cdots \longrightarrow R_{2}^{j} \stackrel{d}{\longrightarrow} R_{1}^{j} \stackrel{d}{\longrightarrow} R_{0}^{j} \stackrel{\epsilon}{\longrightarrow} A^{j} \longrightarrow 0
$$

should be exact.

$(i v)$ If one forgets the differential $d, R$ should be a polynomial (super)algebra in homogeneous generators. We suppose that in each polynomial degree one has a finite number of generators.

Semi-free resolutions exist for each $A$ but they are not unique (sometimes they are called Tate resolutions).

Example 1.1. Let us consider the Koszul complex

$$
K\left(R_{0} ; \mathbf{f}\right):=k\left[x_{0}, \ldots, x_{N}\right] \otimes \Lambda\left\langle\xi_{1} \cdots \xi_{M}\right\rangle,
$$

where $\Lambda$ denotes the exterior algebra; the differential is defined by the formula $d \xi_{j}=f_{j}$. The requirements $(i),(i i),(i v)$ are fulfilled (the homological degree of $x_{i}$ is 1 ). The requirement (iii) holds iff the sequence $\mathbf{f}=\left\{f_{1}, \ldots, f_{M}\right\}$ is regular.

If this is the case, the Krull dimension of $A$ is given by a simple formula

$$
\operatorname{dim} A=N+1-M,
$$

where $N+1$ is the number of $x_{i}$ and $M$ is the number of $\xi_{j}$; one can say that $N+1-M$ is the "superdimension" of the polynomial ring $k\left[x_{0}, \ldots, x_{N}\right] \otimes \Lambda\left\langle\xi_{1} \cdots \xi_{M}\right\rangle$ (the number of even generators minus the number of odd generators).

In general one can consider a semi-free resolution of $A$ as a natural replacement of the Koszul complex. One of the main goals of this paper is to propose an analog of the equation (1.1) valid for not necessarily complete intersections.

If our algebra $A$ is not a complete intersection then a semi-free resolution will be infinite. However, one can associate with such a resolution a sequence of integer numbers and to show that $\operatorname{dim} A$ can be written as a "sum" of these numbers; this will be the required generalization of (1.1). One puts the sum inside of the quotation marks since we have here a divergent series, the sum of which is calculated be means of a regularization, following a classical procedure of Riemann.

In fact, our formula is nothing else but the Mellin transform of the classical theorem by Hilbert which says that $\operatorname{dim} A$ is equal to the order of pole of the Hilbert series of $A$ at 1 . Moreover, we give a similar interpretation for the other numerical invariants of $A$ such as the degree. For details, see Sections 2.1-2.4.

Except for the case of the complete intersection which is rather seldom, there exists a wider class of algebras which admit a remarkable explicit semi-free resolution. Namely, if $A$ is a Koszul algebra then one can take for $R$ the Chevalley cochain complex of the graded Lie algebra $L$ Koszul dual to $A$; one can call it the Koszul-Chevalley resolution, cf. Section 3.4 for details.

As has been noted by many the notion of the Koszul duality is of fundamental importance in Physics. For us a motivating reference is the work of M. Movshev and A. Schwarz. It turns out it is closely related to the "gauge fields-strings duality" dear to Polyakov, cf. [28].

In the second part of this article we discuss a (probably) simplest nontrivial case of a Koszul algebra: we take for $A$ the homogeneous ring of the Veronese curve $X=\mathbb{P}^{1} \hookrightarrow \mathbb{P}^{N}$. This part 
contains no new results; rather it is a review of some classical and modern theorems related to the Koszul-Chevalley resolution of $A$. This topic turns out remarkably rich; it revolves around the Gauss cyclotomic identity. We see here Euler products, Witt theorem, Polya theory and a formula of Polyakov. A particular case of a deep theorem by Kempf and Bezrukavnikov says that $A$ is a Koszul algebra. We will see in Theorem 3.17 that the "numerical" manifestation of this fact is precisely the Gauss cyclotomic identity.

1.2. At the end of this introduction let us say a few words about the algebras interesting to the physicists; we hope to return to these questions later on. In his seminal papers N. Berkovits considers the algebra of functions on the space of pure spinors of dimension 10, the quotient of the polynomial ring in 16 variables $R_{0}=\mathbb{C}\left[\lambda^{1}, \ldots, \lambda^{16}\right]$ by the ideal generated by 10 quadratic elements

$$
\sum_{\alpha, \beta} \lambda^{\alpha} \gamma_{\alpha \beta}^{m} \lambda^{\beta}, \quad m=0, \ldots, 9
$$

The corresponding projective variety $i: X=\operatorname{Proj} A \hookrightarrow \mathbb{P}^{15}$ is the hermitian symmetric space $X=S O(10, \mathbb{C}) / U(5)$. The canonical bundle is $\omega_{X}=\mathcal{O}_{X}(-8)$ where $\mathcal{O}_{X}(1)=i^{*} O_{\mathbb{P}^{15}}$, cf. [13].

The Hilbert series of $A$ is equal to

$$
H(A ; t)=\frac{1-10 t^{2}+16 t^{3}-16 t^{5}+10 t^{6}-t^{8}}{(1-t)^{16}}=\frac{(1+t)\left(1+4 t+t^{2}\right)}{(1-t)^{11}} .
$$

The algebra $A$ is $\operatorname{Koszul}^{1}$.

Let $L=\bigoplus_{i=1}^{\infty} L_{i}$ be the graded Lie algebra Koszul dual to $A$ and $C^{*}(L)$ its Chevalley cochain complex - the Koszul-Chevalley resolution of $A$. As a graded algebra it is the tensor product

$$
C^{*}(L)=S\left(L[1]^{*}\right)=S L_{1}^{*} \otimes \Lambda L_{2}^{*} \otimes S L_{3}^{*} \otimes \cdots
$$

(more precisely the inductive limit of finite products; here $S$ denotes the symmetric algebra and the star denotes the dual space). Let us consider a graded commutative algebra

$$
\mathcal{C}_{1}(L)=C^{*}(L) \otimes \Lambda L_{1}^{*}
$$

One can define a differential on this algebra that will make a dga whose cohomology will be

$$
H^{*}\left(\mathcal{C}_{1}(L)\right)=\operatorname{Tor}_{*}^{R_{0}}(A, \mathbb{C})=: Q .
$$

The dga $C_{1}(L)$ is quasi-isomorphic to the Berkovits algebra studied by Movshev and Schwarz, cf. [24].

The graded commutative algebra $Q$ is called the algebra of syzygies. For $i \geq 1$ denote by $L_{\geq i} \subset L$ the graded Lie subalgebra $\oplus L_{j}$. It follows from the above description that $Q$ is isomorphic to the algebra $H^{*}\left(L_{\geq 2} ; \mathbb{C}\right)^{j \geq i}$ - this is a result contained in [13].

Generalizing this construction, consider for each $i \geq 1$ a graded commutative algebra

$$
\mathcal{C}_{i}(L)=C^{*}(L) \otimes \Lambda L_{1}^{*} \otimes S L_{2}^{*} \otimes \cdots \otimes F_{i} L_{i}^{*},
$$

where $F_{i}=S$ if $i$ is even and $\Lambda$ otherwise. One expects that it is possible to introduce a differential on $\mathcal{C}_{i}(L)$ which provides a dga whose cohomology is $H^{*}\left(L_{\geq i}\right)$. For example, an algebra quasi-isomorphic to $\mathcal{C}_{3}(L)$ has been studied in a very interesting paper [1].

\footnotetext{
${ }^{1}$ The Kempf-Bezrukavnikov theorem says that the coordinate rings of homogeneous spaces of the form $G / P$ where $G$ is semisimple complex and $P$ is a parabolic, are Koszul. Another manifestation of this is the Kapranov's description (à la Beilinson and Bernstein-Gelfand-Gelfand) of the derived category of coherent sheaves on $G / P$.
} 
The algebra of syzygies $Q$ is a remarkable object. It is finite-dimensional over $\mathbb{C}, Q=\underset{i=0}{\oplus} Q_{i}$ and admits, after [13] a scalar product $Q_{i} \otimes Q_{3-i} \longrightarrow \mathbb{C}$ compatible with the multiplication. One can imagine $Q$ as the cohomology of a smooth compact oriented variety of dimension 3 .

A few words about the aim of the paper and the novelty of the results. The physicists are interested in quantum strings fluctuating on the singular space $\operatorname{Spec}(A)$. To pass to strings one has to study the chiral analogs of the above algebras, cf. [1, 3]. The construction of the chiral analog of such algebras, the chiralization, can be accomplished with the use of free infinite resolutions of the above algebras. In this paper we put together the properties of Koszul algebras and their infinite resolutions which contribute to the properties of the chiral algebras [1,3]. We introduce and study in Part I the regularization technique following the insight from [5] which allows to define a part of the above chiral algebra structure. The Part II contains samples of calculations with the equivariant Hilbert series which one needs to define the action of an appropriate Kac-Moody on the chiralization of the above algebras. We are planning to return to chiralization of these objects in a separate publication.

Otherwise, one can regard this topic from the point of view of the string topology of Chas and Sullivan. The string theory is the study of spaces of loops. Let $M$ be a closed oriented manifold of dimension $d, \Omega M$ its free loop space. A fundamental theorem of Chas and Sullivan, cf. [8], says that the homology of $M$ shifted by $d$

$$
\mathbb{H}_{*}(\Omega M):=H_{*+d}(\Omega M)
$$

admits a structure of a Batalin-Vilkovisky (BV) algebra. If $M$ is simply connected then $\mathbb{H}_{*}(\Omega M)$ is isomorphic to the Hochschild cohomology

$$
\mathbb{H}_{*}(\Omega M)=H H^{*}\left(C^{*}(M), C^{*}(M)\right),
$$

where $C^{*}(M)$ is the complex of singular cochains of $M$, cf. $[7,21]$.

An algebraic counterpart of the Chas-Sullivan theory is the following remarkable result ("the cyclic Deligne conjecture"), cf. $[18,32]$ : let $A$ be an associative algebra with an invariant scalar product; then the complex of Hochschild cochains $C H^{*}(A)$ admits a structure of a homotopy BV algebra.

Returning to the Berkovits algebras $\mathcal{C}_{1}(L), Q$, cf. (1.2), (1.3), it would be very interesting to study their Hochschild (as well as cyclic) cohomology. In view of the previous remarks, it should be closely related to the state space of the Berkovits string ${ }^{2}$.

It is worth mentioning certain analogy between the "chiral" and "topological" points of view. For example the Deligne conjecture: the Hochschild cochain complex $C H^{*}(A)$ of an associative algebra $A$ is a homotopy Gerstenhaber algebra (or more precisely, an algebra over the operad $e_{2}$ of chains of little discs), resembles the Lian-Zuckerman conjecture [20]: the space of a topological (i.e. $N=2$ supersymmetric) vertex algebra is a homotopy Gerstenhaber algebra. If an associative algebra is equipped with an invariant scalar product then $C H^{*}(A)$ becomes a homotopy BV algebra. Which complementary structure one needs on a topological vertex algebra to become a homotopy BV algebra? In other words, does there exist a vertex counterpart of the cyclic Deligne conjecture?

\footnotetext{
${ }^{2}$ It seems that the Polyakov's gauge fields-strings correspondence translates in algebra into the assertion: the Hochschild cohomologies of Koszul dual algebras are isomorphic
} 


\section{First part}

\section{Numerical invariants and regularization}

"Les séries divergentes sont en général quelque chose de bien fatal, et c'est une honte qu'on ose y fonder aucune démonstration. On peut démontrer tout ce qu'on veut en les employant..."

N.-H. Abel, a letter to Holmboe, January 16, 1826

\subsection{Semi-free resolutions and Hilbert series}

2.1.1. Let

$$
A=R_{0} /\left(f_{1}, \ldots, f_{M}\right)=\bigoplus_{i=0}^{\infty} A^{i}
$$

and $i: X:=\operatorname{Proj} A \hookrightarrow \mathbb{P}^{N}:=\operatorname{Proj} R_{0}$ be as in the Introduction; denote by $\mathcal{L}=i^{*} \mathcal{O}_{\mathbb{P}^{N}}(1)$ the corresponding very ample line bundle.

Set $h_{i}=\operatorname{dim}_{k} A^{i}$ and let

$$
H(A ; t)=\sum_{i=0}^{\infty} h_{i} t^{i}
$$

be the Hilbert series; we suppose $X$ to be connected, so $H(A ; 0)=h_{0}=1$. Let

$$
R: \cdots \longrightarrow R_{2} \longrightarrow R_{1} \longrightarrow R_{0} \longrightarrow A \longrightarrow 0
$$

be a semi-free resolution of $A$. For $R_{1}$ one can take $R_{1}=\underset{l=1}{M} R_{0} \xi_{l}$, where the variables $\xi_{l}$ are odd and $d \xi_{l}=f_{l}$, so $\xi_{l} \in R_{1}^{d_{i}}$.

Example 2.1. Suppose that $f_{1}, \ldots, f_{M}$ is a regular sequence, that is, $X$ is a complete intersection. Then one can take for $R$ the Koszul complex, $R_{i}=R_{0} \otimes \Lambda^{i} W$, where $W=\underset{l=1}{\bigoplus} k \xi_{l}$.

The exact sequence (2.1) immediately gives an expression of the Hilbert series:

$$
H(A ; t)=\frac{\prod_{l=1}^{M}\left(1-t^{d_{l}}\right)}{(1-t)^{N+1}}=\frac{\prod_{l=1}^{M}\left(1+t+\cdots+t^{d_{l}-1}\right)}{(1-t)^{d+1}}=\frac{P(t)}{(1-t)^{d+1}}
$$

where

$$
d=N-M=\operatorname{dim} X .
$$

One notes that

$$
\operatorname{deg} X=\prod_{l=1}^{M} d_{l}=P(1)
$$


2.1.2. Let us return to the general case. Recall that $R$ as an algebra is a polynomial superalgebra in homogeneous variables. Let us define integer numbers $a_{n}=$ the number of even generators of polynomial degree $n$ minus the number of odd generators of polynomial degree $n$.

Then the exact sequence (2.1) gives a product expression

$$
H(t)=H(A ; t)=\prod_{n=1}^{\infty}\left(1-t^{n}\right)^{-a_{n}}
$$

similar to $(2.2)$.

This formula shows in particular that the numbers $a_{n}$ depend only on $A$ but not on the resolution $R$.

Similarly to (2.3) and (2.4) we want to show that

$$
\begin{aligned}
& \operatorname{dim} X+1=" a_{1}+a_{2}+a_{3}+\cdots ", \\
& \log (\operatorname{deg} X)=-"(\log 1) \cdot a_{1}+(\log 2) \cdot a_{2}+(\log 3) \cdot a_{3}+\cdots " .
\end{aligned}
$$

It is natural also to consider the "higher moments":

$$
" \sum_{n=1}^{\infty} n^{l} a_{n} ", \quad l \geq 1
$$

However, the series at the right hand side are divergent, so one puts the sums in the quotation marks. Our aim will be to perform the summation of these series ${ }^{3}$. There are several ways of doing the summation.

For example, one can write a Lambert series

$$
f(t)=\sum_{n=1}^{\infty} a_{n} \cdot \frac{n t e^{-n t}}{1-e^{-n t}},
$$

cf. [14, App. IV, (1.1)].

This series diverges if $|t|$ is small; but one can show that if $|t|$ is sufficiently big then the series absolutely converges and $t^{-1} f(t)$ is a rational function of $y=e^{-t}$. Therefore one extend $f(t)$ to the complex plane; this function will be holomorphic at $t=0$ (i.e. and $y=1$ ). It is natural to define

$$
" \sum a_{n} ":=f(0)=\underset{t=0}{\operatorname{res}} \frac{f(t)}{t} .
$$

The identity (2.6) will hold true. Moreover, the higher moments (2.8) may be expressed in terms of the coefficients of the Taylor series of $f(t)$ and 0 ; this is noted in [5].

Another classical way of regularization is using the Mellin transform and working with the Dirichlet series. This is what we are going to do.

\subsection{Möbius inversion}

2.2.1. Take the logarithm of (2.5):

$$
\log H(t)=-\sum_{n=1}^{\infty} a_{n} \log \left(1-t^{n}\right)=\sum_{n=1}^{\infty} \sum_{l=1}^{\infty} a_{n} \frac{t^{l n}}{k}=\sum_{m=1}^{\infty}\left(\sum_{l \mid m} \frac{a_{l}}{m / l}\right) \cdot t^{m}
$$

\footnotetext{
${ }^{3}$ The classical sources about divergent series are the books [14, 31].
} 
and then the derivative:

$$
\frac{t H^{\prime}(t)}{H(t)}=\sum_{n=1}^{\infty} \frac{n a_{n} t^{n}}{1-t^{n}}=\sum_{m=1}^{\infty}\left(\sum_{l \mid m} l a_{l}\right) \cdot t^{m} .
$$

In other words, if one denotes

$$
\frac{t H^{\prime}(t)}{H(t)}=\sum_{m=1}^{\infty} b_{m} t^{m}
$$

then

$$
b_{m}=\sum_{l \mid m} l a_{l} .
$$

2.2.2. Recall that the Möbius function $\mu: \mathbb{N}_{+}=\{1,2, \ldots\} \longrightarrow\{-1,0,1\}$ is defined by: $\mu(1)=1, \mu(n)=(-1)^{l}$ if $n=p_{1} p_{2} \cdots p_{l}$ is a product of $l$ distinct prime numbers, and $\mu(n)=0$ if $n$ contains squares.

Another definition is by a generating series: if one defines the Riemann $\zeta$ function by the Euler product

$$
\zeta(s)=\sum_{n=1}^{\infty} n^{-s}=\prod_{p \text { prime }}\left(1-p^{-s}\right)^{-1},
$$

then

$$
\zeta(s)^{-1}=\prod_{p \text { prime }}\left(1-p^{-s}\right)=\sum_{n=1}^{\infty} \mu(n) n^{-s} .
$$

The Möbius inversion formula says that if $f: \mathbb{N}_{+} \longrightarrow \mathbb{C}$ is a function and if a function $g: \mathbb{N}_{+} \longrightarrow \mathbb{C}$ is defined by

$$
g(m)=\sum_{l \mid m} f(l)
$$

then

$$
f(m)=\sum_{l \mid m} \mu(m / l) g(l) .
$$

Applying this formula to the function $f(m)=m a_{m}$, we get

$$
m a_{m}=\sum_{l \mid m} \mu(m / l) b_{l} .
$$

\subsection{Dirichlet series}

2.3.1. Let $P(t)$ be a polynomial with complex coefficients such that $P(0)=1$, so one can write

$$
P(t)=\prod_{l=1}^{p}\left(1-\alpha_{l} t\right) .
$$


Consider the product (2.5) for $P(t)$ :

$$
P(t)=\prod_{n=1}^{\infty}\left(1-t^{n}\right)^{-a_{n}} .
$$

So if

$$
\frac{t P^{\prime}(t)}{P(t)}=\sum_{m=1}^{\infty} b_{m} t^{m}
$$

then

$$
m a_{m}=\sum_{l \mid m} \mu(m / l) b_{l} .
$$

On the other hand

$$
\frac{t P^{\prime}(t)}{P(t)}=-\sum_{r=1}^{p} \frac{\alpha_{r} t}{1-\alpha_{r} t}=-\sum_{r=1}^{p} \sum_{m=1}^{\infty} \alpha_{r}^{m} t^{m},
$$

i.e.

$$
b_{m}=-\sum_{r=1}^{p} \alpha_{r}^{m}
$$

wherefrom

$$
a_{m}=-m^{-1} \sum_{r=1}^{p} \sum_{l \mid m} \mu(m / l) \alpha_{r}^{l} .
$$

One puts

$$
\rho(P)=\max _{r}\left|\alpha_{r}\right| .
$$

It follows that if $\rho(P)>1$, then $\left|a_{m}\right|$ grows as fast as $m^{-1} \rho(P)^{m}$.

2.3.2. Let us consider the Dirichlet series

$$
\sum_{n=1}^{\infty} a_{n} n^{-s}
$$

We see that if $\rho(P) \leq 1$ then this series absolutely converges for $\mathfrak{R e}(s)>1$, and if $\rho(P)>1$, it diverges for all $s$; in this case (which in fact is interesting to us) one has to do something else.

Let us write formally with [5]:

$$
\begin{aligned}
-\sum_{n=1}^{\infty} a_{n} n^{-s} & =\sum_{n=1}^{\infty} \sum_{r=1}^{p} \sum_{l \mid n} \mu(n / l) \alpha_{r}^{l} n^{-1-s}=\sum_{r=1}^{p} \sum_{l, m=1}^{\infty} \mu(m) \alpha_{r}^{l}(l m)^{-1-s} \\
& =\sum_{m=1}^{\infty} \mu(m) m^{-1-s} \cdot \sum_{r=1}^{p} \sum_{l=1}^{\infty} \alpha_{r}^{l} l^{-1-s}=\zeta(s+1)^{-1} \sum_{r=1}^{p} \sum_{l=1}^{\infty} \alpha_{r}^{l} l^{-1-s} .
\end{aligned}
$$

Now if we rewrite, after Riemann,

$$
l^{-1-s}=\frac{1}{\Gamma(s+1)} \int_{0}^{\infty} e^{-l t} t^{s} d t
$$


wherefrom

$$
\sum_{l=1}^{\infty} \alpha_{r}^{l} l^{-1-s}=\frac{1}{\Gamma(s+1)} \int_{0}^{\infty} \sum_{l=1}^{\infty} \alpha_{r}^{l} e^{-l t} t^{s} d t=\frac{1}{\Gamma(s+1)} \int_{0}^{\infty} \frac{\alpha_{r} e^{-t} t^{s}}{1-\alpha_{r} e^{-t}} d t
$$

(justified if $\left|\alpha_{r}\right|<1$ ). Because

$$
\frac{e^{-t}}{1-\alpha_{r} e^{-t}}=\frac{1}{e^{t}-\alpha_{r}}
$$

the integral

$$
\int_{0}^{\infty} \frac{\alpha_{r} e^{-t} t^{s}}{1-\alpha_{r} e^{-t}} d t
$$

absolutely converges for $\mathfrak{R e}(s)>-1$ if $\alpha_{r} \notin[0,1]$ (since $P(1)=1$, one has $\alpha_{r} \neq 0$ automatically). One notes that

$$
\sum_{r=1}^{p} \frac{\alpha_{r} e^{-t}}{1-\alpha_{r} e^{-t}}=-\frac{e^{-t} P^{\prime}\left(e^{-t}\right)}{P\left(e^{-t}\right)} .
$$

On the other hand, let us write the functional equation for $\zeta(s)$ (cf. $[34,13.151])$ :

$$
2^{1-s} \Gamma(s) \zeta(s) \cos (\pi s / 2)=\pi^{s} \zeta(1-s)
$$

and replace $s$ by $s+1$ :

$$
2^{-s} \Gamma(s+1) \zeta(s+1) \cos (\pi(s+1) / 2)=\pi^{s+1} \zeta(-s),
$$

i.e.

$$
\frac{1}{\Gamma(s+1) \zeta(s+1)}=-2^{-s} \pi^{-s-1} \sin (\pi s / 2) \zeta(-s)^{-1} .
$$

It follows:

$$
\begin{aligned}
\sum_{n=1}^{\infty} a_{n} n^{-s} & =\frac{1}{\Gamma(s+1) \zeta(s+1)} \int_{0}^{\infty} \frac{e^{-t} P^{\prime}\left(e^{-t}\right)}{P\left(e^{-t}\right)} t^{s} d t \\
& =-\frac{2^{-s} \pi^{-s-1} \sin (\pi s / 2)}{\zeta(-s)} \int_{0}^{\infty} \frac{e^{-t} P^{\prime}\left(e^{-t}\right)}{P\left(e^{-t}\right)} t^{s} d t
\end{aligned}
$$

2.3.3. Consider the last integral

$$
I_{P}(s):=\int_{0}^{\infty} \frac{e^{-t} P^{\prime}\left(e^{-t}\right)}{P\left(e^{-t}\right)} t^{s} d t .
$$

It is well defined and represents a holomorphic function of $s$ on the half-plane $\mathfrak{R e}(s)>-1$ as soon as the condition (2.19) below is verified:

The roots of $P(t)$ do not lie in the segment $[0,1]$.

As it was kindly pointed out by one of the referees the condition (2.19) holds. Indeed, since the algebra $A$ is affine, it has polynomial growth, so that the radius of convergence of the series $H(A, t)$ is $0 \leq t<1$. Because the coefficients of the formal power series $H(A, t)$ are nonnegative, we have $H(A, t)>0$ for all $0 \leq t<1$. It follows that $P(A, t)>0$ with $H(A, t)$. Also, the is function $H(A, t)$ is rational (Hilbert-Serre), so that it has a pole at $t=1$ of the order $d+1$. This means that the value $P(A, 1)$ is nonzero as well. 
2.3.4. Following Riemann and using the notations of [34], consider the integral

$$
J_{P}(s)=\int_{\infty}^{(0+)} \frac{e^{-t} P^{\prime}\left(e^{-t}\right)}{P\left(e^{-t}\right)}(-t)^{s} d t
$$

where $\int_{\infty}^{(0+)}$ denotes the integral along the contour

$$
\begin{aligned}
C= & \{t=a+\epsilon i, \infty>a \geq \epsilon\} \cup\left\{t=\sqrt{2} \epsilon e^{i \theta}, \pi / 4 \leq \theta \leq 7 \pi / 4\right\} \\
& \cup\{t=a-\epsilon i, \epsilon \leq a<\infty\},
\end{aligned}
$$

where $\epsilon>0$ is sufficiently small. Here $(-t)^{s}=e^{s \log (-t)}$, and we use the branch of the logarithm $\pi \geq \arg t \geq-\pi$ on the small circle. Then

$$
J_{P}(s)=2 i \sin (\pi s) I_{P}(s)
$$

cf. [34, 12.22]. But $J_{P}(s)$ is an entire function. Therefore, if one sets

$$
z_{P}(s)=-\frac{2^{-s} \pi^{-s-1} \sin (\pi s / 2)}{\zeta(-s)} \cdot \frac{1}{2 i \sin (\pi s)} J_{P}(s)=\frac{i \cdot 2^{-s-2} \pi^{-s-1}}{\cos (\pi s / 2) \zeta(-s)} J_{P}(s),
$$

this defines the analytic continuation of (2.20) to a meromorphic function on the complex plane.

2.3.5. Let us return to the situation of Section 2.1; it is known that the Hilbert series is a rational function of the form

$$
H(A ; t)=\frac{P(A ; t)}{(1-t)^{d+1}},
$$

where $d=\operatorname{dim} X=\operatorname{dim} A-1$ and $P(A ; t)$ is a polynomial with integer coefficients (cf. a simple Example 2.2). One has $P(A ; 0)=H(A ; 0)=1$ since we suppose that $X$ connected.

In view of the preceding discussion we define

$$
z(X, \mathcal{L} ; s)=\frac{1}{\Gamma(s+1) \zeta(s+1)} \int_{0}^{\infty} \frac{e^{-t} H^{\prime}\left(A ; e^{-t}\right)}{H\left(A ; e^{-t}\right)} t^{s} d t=d+1+z_{P}(s)
$$

(because $\left.\Gamma(s+1) \zeta(s+1)=\int_{0}^{\infty}\left(t^{s} /\left(e^{t}-1\right)\right) d t\right)$. So if

$$
H(A ; t)=\prod_{n=1}^{\infty}\left(1-t^{n}\right)^{-A_{n}},
$$

then

$$
z(X, \mathcal{L} ; s)=" \sum_{n=1}^{\infty} A_{n} n^{-s "},
$$

the quotation marks mean that the sum is regularized. We define "the Dirichlet summation":

$$
\text { " } \sum_{n=1}^{\infty} A_{n} ":=z(X, \mathcal{L} ; 0),
$$

and

$$
\text { " } \sum_{n=1}^{\infty} \log n \cdot A_{n} ":=-z^{\prime}(X, \mathcal{L} ; 0),
$$


2.3.6. We have $\zeta(0)=-1 / 2$ and $\zeta^{\prime}(0)=-\log \sqrt{2 \pi} \neq 0$ (cf. [33, Ch. VII, $\left.\S 9\right]$ ). The integral $I_{P}(0)$ is well defined (below we shall compute its value), so the formula $(2.23)$ gives $z_{P}(0)=0$, wherefrom

$$
z(X, \mathcal{L} ; 0)=d+1
$$

cf. (2.6). On the other hand, $z^{\prime}(X, \mathcal{L} ; s)=z_{P}^{\prime}(s)$, and a unique term giving a nontrivial contribution into $z^{\prime}(X, \mathcal{L} ; 0)$, is

$$
-\left.\left(\frac{2^{-s} \pi^{-s-1} \cdot(\pi / 2) \cos (\pi s / 2)}{\zeta(-s)}\right)\right|_{s=0} \cdot I_{P}(0)
$$

The first factor gives 1 , whereas

$$
\begin{aligned}
I_{P}(0) & =\int_{0}^{\infty} \frac{e^{-t} P^{\prime}\left(e^{-t}\right)}{P\left(e^{-t}\right)} d t=-\int_{0}^{\infty} \frac{d \log P\left(e^{-t}\right)}{d t} d t \\
& =-\left.\log P\left(e^{-t}\right)\right|_{0} ^{\infty}=-\log P(0)+\log P(1)=\log P(1),
\end{aligned}
$$

wherefrom

$$
z^{\prime}(X, \mathcal{L} ; 0)=\log P(1) .
$$

But it is known that $P(1)=\operatorname{deg} X$, which implies

$$
\operatorname{deg} X=e^{z^{\prime}(X, \mathcal{L} ; 0)},
$$

cf. (2.7).

Example 2.2 (Veronese curve and the Weil zeta function). Let $X=\mathbb{P}^{1}, \mathcal{L}=\mathcal{O}(q+1)$, $q \geq 1$. Then $A^{n}=\Gamma(X, \mathcal{O}((q+1) n)$, which implies

$$
H(A ; t)=\sum_{n=0}^{\infty}((q+1) n+1) t^{n}=\frac{1+q t}{(1-t)^{2}},
$$

and

$$
P(t)=1+q t=\prod_{m=1}^{\infty}\left(1-t^{m}\right)^{p_{m}(-q)},
$$

where

$$
p_{m}(x)=\frac{1}{m} \sum_{l \mid m} \mu(m / l) x^{l} .
$$

It is well known that if $q$ is a prime power then $p_{m}(q)$ is equal to the number of monic irreducible polynomials of degree $m$ in $\mathbb{F}_{q}[T]$. In fact, we have

$$
Z_{\mathbb{F}_{q}}\left(\mathbb{A}^{1}, t\right)=\frac{1}{P(-t)}=\prod_{m=1}^{\infty}\left(1-t^{m}\right)^{-p_{m}(q)}
$$

- this is the Euler product of the zeta function of the affine line over the finite field $\mathbb{F}_{q}$.

(The idea that the Hilbert series of a variety is equal to the Weil zeta function of another one seems very strange. Cf. [12] however ${ }^{4}$.)

\footnotetext{
${ }^{4}$ We are grateful to Yu.I. Manin who showed us this very interesting paper.
} 


\subsection{Values at negative points}

2.4.1. Let $s=-m$ be a negative integer. In this case one can close the contour in the integral (2.20):

$$
J_{P}(-m)=(-1)^{m} \int_{|t|=\epsilon} Q(t) t^{-m} d t=2 \pi i \cdot(-1)^{m} \underset{t=0}{\operatorname{res}} Q(t) t^{-m},
$$

where

$$
Q(t)=\frac{e^{-t} P^{\prime}\left(e^{-t}\right)}{P\left(e^{-t}\right)}=-\frac{d \log P\left(e^{-t}\right)}{d t} .
$$

So if $Q(t)=q_{0}+q_{1} t / 1 !+q_{2} t^{2} / 2 !+\cdots$ is the Taylor series at 0 then

$$
J_{P}(-m-1)=2 \pi i \cdot(-1)^{m+1} \frac{q_{m}}{m !} .
$$

Here are some first values:

$$
\begin{aligned}
& q_{0}=\frac{P^{\prime}(1)}{P(1)}, \quad q_{1}=-q_{0}+q_{0}^{2}-\frac{P^{\prime \prime}(1)}{P(1)}, \\
& q_{2}=q_{0}-3 q_{0}^{2}+2 q_{0}^{3}+3\left(1-q_{0}\right) \frac{P^{\prime \prime}(1)}{P(1)}+\frac{P^{\prime \prime \prime}(1)}{P(1)} .
\end{aligned}
$$

2.4.2. One can say this in a different way: if

$$
P(t)=1+c_{1} t+\cdots+c_{D} t^{D}
$$

and we imagine the numbers $c_{i}$ as "Chern classes" then $q_{m}$ will be the coefficients of the logarithm of the Todd genus ...

2.4.3. Now applying (2.22): for $m=1$, the function $\zeta(s)$ has a simple pole at $s=1$ with the residue 1 , whence

$$
z_{P}(-1)=\frac{2 P^{\prime}(1)}{P(1)}
$$

Remark 2.3. Suppose that $P(t)$ is a "reciprocal" polynomial, i.e.

$$
t^{\operatorname{deg} P} P(1 / t)=P(t),
$$

which happens rather often. In this case it is easy to see that

$$
\frac{2 P^{\prime}(1)}{P(1)}=\operatorname{deg} P
$$

so $z_{P}(-1)=\operatorname{deg} P$.

2.4.4. For $m>1$ one has to distinguish two cases.

(a) If $m=2 l$ is even then $\cos (\pi l)=(-1)^{l}$, and after Euler,

$$
\zeta(2 l)=(-1)^{l-1} \frac{(2 \pi)^{2 l}}{2(2 l) !} b_{2 l},
$$


where the Bernoulli numbers are defined by the generating series

$$
\frac{S}{e^{S}-1}=1-\frac{S}{2}+\sum_{l=1}^{\infty} \frac{b_{2 l}}{(2 l) !} S^{2 l}
$$

Here are some first values:

$$
b_{2}=\frac{1}{6}, \quad b_{4}=-\frac{1}{30}, \quad b_{6}=\frac{1}{42}, \quad b_{8}=-\frac{1}{30} .
$$

It follows:

$$
z_{P}(-2 l)=\frac{2 l q_{2 l-1}}{b_{2 l}}
$$

So if the numbers $a_{n}$ are defined by (2.24) then

$$
" \sum_{n=1}^{\infty} n^{2 l} a_{n} "=z(X, \mathcal{L} ;-2 l)=d+1+\frac{2 l q_{2 l-1}}{b_{2 l}}
$$

- a formula found empirically in [5, (4.21)].

(b) If $m=2 l+1, l>0$ then $z_{P}(s)$ has a simple pole at $s=-m$, with the residue

$$
\underset{s=-2 l-1}{\operatorname{res}} z_{P}(s)=(-1)^{l} \frac{(2 \pi)^{2 l} q_{2 l}}{(2 l+1) ! \zeta(2 l+1)} \text {. }
$$

\subsubsection{Hurwitz zeta function.}

(a) We have $J_{P}(s)=\sum_{r=1}^{p} J_{\alpha_{r}}(s)$ where

$$
J_{\alpha}(s)=-\int_{\infty}^{(0+)} \frac{\alpha}{e^{t}-\alpha}(-t)^{s} d t
$$

Let us choose a value $\beta=\log \alpha$. The expression under the integral $f_{\alpha}(t, s)=\left(\alpha / e^{t}-\alpha\right) \cdot(-t)^{s}$ has the poles at points $t_{n}=\beta+2 \pi i n, n \in \mathbb{Z}$. By the Cauchy formula,

$$
J_{\alpha}(s)+2 \pi i \sum_{n=-\infty}^{\infty} \operatorname{res}_{t=t_{n}} f_{\alpha}(t, s)=0
$$

if $\mathfrak{R e}(s)<-1$, then

$$
J_{\alpha}(s)=2 \pi i \sum_{n=-\infty}^{\infty}(-\beta-2 \pi i n)^{s}
$$

which gives $J_{P}(s)$ in the form of a series absolutely convergent for $\mathfrak{R e}(s)<-1$, expressible in terms of the Hurwitz zeta functions. 
(b)

Example 2.4. Take $P(t)=1+p t$, where $p \in \mathbb{Z}_{>0}$, cf. Example 2.2. Then $q_{0}=p /(p+1)$, $q_{1}=-p /(p+1)^{2}$ (cf. Section 2.4.1), so

$$
\frac{J_{P}(-2)}{2 \pi i}=-\frac{p}{(p+1)^{2}}
$$

On the other hand, take $\beta=\log (-p)=\log p+\pi i$, and the above series will be written as

$$
\sum_{n=-\infty}^{\infty}(-\log p-\pi i-2 \pi i n)^{-2}=\sum_{n=0}^{\infty}\left\{(-\log p+(2 n+1) \pi i)^{-2}+(-\log p-(2 n+1) \pi i)^{-2}\right\}
$$

so one came to an identity

$$
\sum_{n=0}^{\infty}\left\{(-\log p+(2 n+1) \pi i)^{-2}+(-\log p-(2 n+1) \pi i)^{-2}\right\}=-\frac{p}{(p+1)^{2}}
$$

For example, for $p=3,-p /(p+1)^{2}=-0,1875$, whereas an approximate value given by MAPLE is

$$
\sum_{n=0}^{1000}\left\{(-\log p+(2 n+1) \pi i)^{-2}+(-\log p-(2 n+1) \pi i)^{-2}\right\}=-0,187449 \ldots ;
$$

we see that the convergence is very slow.

(c) If $s=-1$, the series (2.27) in our example is still "Eisenstein summable", and on gets a rational value:

$$
\sum_{n=0}^{\infty}\left\{(-\log p+(2 n+1) \pi i)^{-1}+(-\log p-(2 n+1) \pi i)^{-1}\right\}=-\frac{2(p-1)}{p+1} .
$$

This is an easy corollary of the decomposition of $\cot z$ into simple fractions ${ }^{5}$. But this value is different from $-q_{0}=-p /(p+1)$ : we have "an anomaly".

2.4.6. Suppose that our variety $X$ is smooth, $H^{i}\left(X, \mathcal{L}^{\otimes n}\right)=0$ for all $i>0$ and $n \geq 0$. Then $A^{n}=\Gamma\left(X, \mathcal{L}^{\otimes n}\right)$, and we can switch on the Riemann-Roch-Hirzebruch [15]:

$$
h_{n}=\operatorname{dim} H^{0}\left(X, \mathcal{L}^{\otimes n}\right)=\int_{X} e^{n c_{1}(\mathcal{L})} \operatorname{Td}\left(\mathcal{T}_{X}\right),
$$

where $\mathcal{T}_{X}$ denotes the tangent bundle, Td the Todd genus, given for line bundle $E$ by the formula,

$$
\operatorname{Td}(E)=\frac{c_{1}(E)}{1-e^{-c_{1}(E)}},
$$

so $\operatorname{Td}\left(\mathcal{T}_{X}\right)=1+\operatorname{Td}_{1}\left(\mathcal{T}_{X}\right)+\operatorname{Td}_{2}\left(\mathcal{T}_{X}\right)+\cdots$, where

$$
\operatorname{Td}_{1}\left(\mathcal{T}_{X}\right)=\frac{c_{1}\left(\mathcal{T}_{X}\right)}{2}, \quad \operatorname{Td}_{2}\left(\mathcal{T}_{X}\right)=\frac{c_{1}^{2}\left(\mathcal{T}_{X}\right)+c_{2}\left(\mathcal{T}_{X}\right)}{12} .
$$

\footnotetext{
${ }^{5}$ We thank Oleg Ogievietsky who has noted this.
} 
One writes: $e^{n c_{1}(\mathcal{L})}=\sum_{i=0}^{\infty} c_{1}(\mathcal{L})^{i} n^{i} / i$ !, so $h_{n}=R(n)$, where

$$
\begin{aligned}
R(t)= & \left(\int_{X} c_{1}(\mathcal{L})^{d}\right) \cdot \frac{t^{d}}{d !}+\left(\int_{X} c_{1}(\mathcal{L})^{d-1} c_{1}\left(\mathcal{T}_{X}\right)\right) \cdot \frac{t^{d-1}}{2(d-1) !}+ \\
& +\left(\int_{X} c_{1}(\mathcal{L})^{d-2}\left(c_{1}\left(\mathcal{T}_{X}\right)^{2}+c_{2}\left(\mathcal{T}_{X}\right)\right)\right) \cdot \frac{t^{d-2}}{12(d-2) !}+\cdots=r_{d} \frac{t^{d}}{d !}+r_{d-1} \frac{t^{d-1}}{(d-1) !}+\cdots
\end{aligned}
$$

is a polynomial of degree $d=\operatorname{dim} X$, the Hilbert polynomial of the $\operatorname{ring} A$; it is a polynomial with rational coefficients which takes integer values at integer argument, whence

$$
R(t)=\sum_{i=0}^{d}(-1)^{d-i} e_{d-i}\left(\begin{array}{c}
t+i \\
i
\end{array}\right),
$$

where $e_{0}, e_{1}, \ldots, e_{d} \in \mathbb{Z}$. For example,

$$
\begin{aligned}
& e_{0}=r_{d}=\int_{X} c_{1}(\mathcal{L})^{d}, \\
& e_{1}=\frac{d+1}{2} r_{d}-r_{d-1}=\frac{1}{2} \int_{X}\left((d+1) c_{1}(\mathcal{L})^{d}-c_{1}(\mathcal{L})^{d-1} c_{1}\left(\mathcal{T}_{X}\right)\right), \\
& e_{2}=r_{d-2}-\frac{d}{2} r_{d-1}+\frac{(d+1)(3 d-2)}{24} r_{d}
\end{aligned}
$$

(we use $\left.\sum_{1 \leq i<j \leq d} i j=d\left(d^{2}-1\right)(3 d+2) / 24\right)$.

On the other hand it is known that

$$
e_{i}=\frac{P^{(i)}(1)}{i !} \text {. }
$$

It follows:

$$
e^{z^{\prime}(X, \mathcal{L} ; 0)}=P(1)=e_{0}=\int_{X} c_{1}(\mathcal{L})^{d},
$$

so one finds the degree of $X$. Next

$$
\begin{aligned}
z_{P}(-1) & =-\frac{2 P^{\prime}(1)}{P(1)}=-\frac{2 e_{1}}{e_{0}}=-\frac{1}{e_{0}} \int_{X}\left((d+1) c_{1}(\mathcal{L})^{d}-c_{1}(\mathcal{L})^{d-1} c_{1}\left(\mathcal{T}_{X}\right)\right) \\
& =-d-1+\frac{1}{e_{0}} \int_{X} c_{1}(\mathcal{L})^{d-1} c_{1}\left(\mathcal{T}_{X}\right)
\end{aligned}
$$

i.e.

$$
z(X, \mathcal{L} ;-1)=\frac{1}{e_{0}} \int_{X} c_{1}(\mathcal{L})^{d-1} c_{1}\left(\mathcal{T}_{X}\right)
$$

etc.

One can put these calculations into a formal "generating function" for the moments

$$
M_{l}=" \sum_{n=1}^{\infty} n^{l} a_{n} "
$$

Note that from the formulas above and using the expansion

$$
\log \left(1-e^{t}\right)=\log (-t)+\frac{t}{2}+\sum_{l=1}^{\infty} \frac{b_{2 l}}{2 l(2 l) !} t^{2 l}
$$


one concludes the formal identity:

$$
-\log P\left(e^{t}\right)=“ \sum_{l=0}^{\infty} \log (l) a_{l}+M_{1} \frac{t}{2}+\sum_{l=1}^{\infty}\left(M_{2 l}\right) \frac{b_{2 l}}{2 l(2 l) !} t^{2 l} " .
$$

The following elegant formula is due to F. Hirzebruch [16]: the polynomial $P(t)$ is a characteristic number defined as

$$
P(t)=\int_{X} \frac{(1-t) e^{(1-t) c_{1}} \widehat{A}\left((1-t)^{2} p_{1}, \ldots,(1-t)^{2 i} p_{2 i}, \ldots\right)}{1-t e^{(1-t) g}},
$$

where $c_{1}, p_{1}, p_{2}, \ldots$ are the first Chern class and the Pontryagin classes of the manifold $X$.

From this it is immediate to express the left hand side of (2.30) as a characteristic number. Indeed

$$
\log P\left(e^{t}\right)=\log \int_{X} \frac{\left(1-e^{t}\right) e^{\left(1-e^{t}\right) c_{1}} \widehat{A}\left(\left(1-e^{t}\right)^{2} p_{1}, \ldots,\left(1-e^{t}\right)^{2 i} p_{2 i}, \ldots\right)}{1-e^{t+\left(1-e^{t}\right)}} .
$$

In order to expand the right hand side into a series of $t$ observe that the constant term of the series under the logarithm is

$$
\int_{X} \frac{1}{1-g}=P(1)
$$

Therefore

$$
\log \frac{1}{\int_{X} \frac{1}{1-g}} \int_{X} \frac{\left(1-e^{t}\right) e^{\left(1-e^{t}\right) c_{1}} \widehat{A}\left(\left(1-e^{t}\right)^{2} p_{1}, \ldots,\left(1-e^{t}\right)^{2 i} p_{2 i}, \ldots\right)}{1-e^{t+\left(1-e^{t}\right)}}
$$

is a series in $t$ equal to $\log P\left(e^{t}\right)-\log P(1)$. This gives the desired generating function.

Example 2.5. Let $X_{g}$ be the moduli space of semi-stable vector bundles of rank 2 with trivial determinant over a Riemann surface of genus $g$; it carries the canonical determinant line bundle $\mathcal{L}_{g}$, cf. [2]. Consider a graded algebra

$$
A_{g}=\bigoplus_{i=0}^{\infty} H^{0}\left(X_{g}, \mathcal{L}_{g}^{\otimes i}\right)
$$

(we thank Peter Zograf who proposed to consider this example).

The coefficients of the Hilbert series $H_{g}(t)=H\left(A_{g} ; t\right)$ can be calculated using the Verlinde formula [2]. Here are some first examples, cf. [36]:

$$
\begin{aligned}
& H_{2}(t)=\frac{1}{(1-t)^{4}}, \quad H_{3}(t)=\frac{1+t+t^{2}+t^{3}}{(1-t)^{7}}=\frac{1-t^{4}}{(1-t)^{8}}, \\
& H_{4}(t)=\frac{1+6 t+21 t^{2}+40 t^{3}+21 t^{4}+6 t^{5}+t^{6}}{(1-t)^{10}}
\end{aligned}
$$

The highest coefficient $r_{d} / d$ ! of the Hilbert polynomial has been calculated in [Wi], who found the value $2 \zeta(2 g-2) /\left(2 \pi^{2}\right)^{g-1}$; here $d=\operatorname{dim} X_{g}=3 g-3$, whence

$$
P_{g}(1)=(-1)^{g} \frac{(3 g-3) !}{(2 g-2) !} b_{2 g-2}=(-1)^{g+1} \frac{(3 g-3) !}{(2 g-1) !} \zeta(-2 g+3)
$$

(one denotes $\left.P_{g}(t)=H_{g}(t)(1-t)^{d+1}\right)$. For example, for $g=4$ one finds $P_{4}(1)=96$, which is compatible with (2.32). 
The above discussion gives a strange expression of this number as a regularized infinite product (starting from $g=4$ ), of the form $\prod_{n=1}^{\infty} n^{a_{n}}$. For example, the beginning of the product for $P_{4}(t)$ will be:

$$
\begin{aligned}
P_{4}(t)= & (1-t)^{-6}\left(1-t^{3}\right)^{16}\left(1-t^{4}\right)^{9}\left(1-t^{5}\right)^{-144}\left(1-t^{6}\right)^{360} \\
& \times\left(1-t^{8}\right)^{-2259}\left(1-t^{9}\right)^{3920} \cdots
\end{aligned}
$$

whence

$$
\zeta(-5)=-\frac{9 !}{7 !} \cdot{ }^{6} 1^{6} 3^{-16} 4^{-9} 5^{144} 6^{-360} 8^{2259} 9^{-3920} \ldots " .
$$

\section{Second part}

\section{Koszulness and infinite resolutions}

\subsection{The Veronese ring}

3.1.1. Let us fix an integer $b \geq 0$, and consider the Veronese embedding

$$
i_{b}: \mathbb{P}^{1} \longrightarrow \mathbb{P}^{b+1}
$$

defined in coordinates by the formula

$$
i_{b}\left(u_{0}: u_{1}\right)=\left(x_{0}: \cdots: x_{b+1}\right):=\left(u_{0}^{b+1}: u_{0}^{b} u_{1}: \cdots: u_{1}^{b+1}\right) .
$$

If $t=u_{1} / u_{0} \in \mathbb{A}^{1} \subset \mathbb{P}^{1}$, then

$$
i_{b}(t)=\left(t, t^{2}, \ldots, t^{b+1}\right) \in \mathbb{A}^{b+1} \subset \mathbb{P}^{b+1} .
$$

The image $X_{b}:=i_{b}\left(\mathbb{P}^{1}\right) \subset \mathbb{P}^{b+1}$ of this embedding is called the Veronese curve (or the moment curve, in view of the last formula).

This curve can be defined in $\mathbb{P}^{b+1}$ by the equations $x_{i} x_{j}-x_{k} x_{l}=0$ if $i+j=k+l$. A minimal system of equations consists of $b(b+1) / 2$ quadratic equations:

$$
f_{i j}:=x_{i} x_{j}-x_{i+1} x_{j-1}=0, \quad 0 \leq i \leq b-1, \quad i+2 \leq j \leq b+1 .
$$

Example 3.1. $i_{0}=\operatorname{Id}_{\mathbb{P}^{1}}$. If $b=1, X_{1} \subset \mathbb{P}^{2}=\left\{\left(x_{0}: x_{1}: x_{2}\right)\right\}$ is defined by one equation:

$$
x_{0} x_{2}-x_{1}^{2}=0 .
$$

The curve $X_{3} \subset \mathbb{P}^{4}$ is defined by 3 equations

$$
x_{0} x_{2}-x_{1}^{2}=0 ; \quad x_{0} x_{3}-x_{1} x_{2}=0 ; \quad x_{1} x_{3}-x_{2}^{2}=0 .
$$

3.1.2. We have $i_{b}^{*} \mathcal{O}_{\mathbb{P}^{b+1}}(1)=\mathcal{O}_{\mathbb{P}^{1}}(b+1)$, all the higher cohomology of this sheaf vanish, and $X_{b}=\operatorname{Spec} \operatorname{Proj} A_{b}$, where

$$
A_{b}=\bigoplus_{n=0}^{\infty} \Gamma\left(\mathbb{P}^{1}, \mathcal{O}((b+1) n)=\mathbb{C}\left[x_{0}, \ldots, x_{b+1}\right] /\left(f_{i j}\right) .\right.
$$

The Hilbert series of the above algebra is as follows:

$$
H\left(A_{b} ; t\right)=\sum_{n=0}^{\infty}((b+1) n+1) t^{n}=\frac{1+b t}{(1-t)^{2}} .
$$


It is not difficult to figure out the $q$-analogue of $(3.1)$ : if we set $[b]_{q}:=\left(q^{b}-q^{-b}\right) /\left(q-q^{-1}\right)$, then:

$$
\begin{aligned}
H_{q}\left(A_{b} ; t\right) & :=\sum_{n=0}^{\infty}[(b+1) n+1]_{q} t^{n}=\left(q-q^{-1}\right)^{-1} \cdot \sum_{n}\left\{q q^{(b+1) n} t^{n}-q^{-1} q^{-(b+1) n} t^{n}\right\} \\
& =\left(q-q^{-1}\right)^{-1} \cdot\left(\frac{q}{1-q^{b+1} t}-\frac{q^{-1}}{1-q^{-b-1} t}\right)=\frac{1+[b]_{q} t}{\left(1-q^{b+1} t\right)\left(1-q^{-b-1} t\right)} .
\end{aligned}
$$

We will use these formulas later in Section 3.4.4.

Example 3.2. For $b=1$ we have,

$$
H\left(A_{1} ; t\right)=\frac{1+t}{(1-t)^{2}}=\frac{1-t^{2}}{(1-t)^{3}},
$$

the ring $A_{1}$ is a cone:

$$
A_{1}=\mathbb{C}\left[x_{0}, x_{1}, x_{2}\right] /\left(x_{0} x_{2}-x_{1}^{2}\right)=B /(f) .
$$

It admits a dga resolution of length 1: the Koszul complex:

$$
K .(B ; f): 0 \longrightarrow B \cdot e \longrightarrow B \longrightarrow A \longrightarrow 0, \quad d(e)=f .
$$

Therefore

$$
K .(B ; f)=B \otimes \Lambda\langle e\rangle=\mathbb{C}\left[x_{0}, x_{1}, x_{2} ; e\right]
$$

as a graded algebra; the homological degree of $x_{i}$ is 0 and of $e$ is 1 . These correspond to the exponents -3 and 1 in (3.3).

There is another interpretation of the Koszul complex. Let $V$ be a vector space $\underset{i=0}{\stackrel{2}{C}} \mathbb{C} \cdot x_{i}$, then $B=S \cdot V$. Consider the dual space $V^{*}=\underset{i=0}{\oplus} \mathbb{C} \cdot y_{i}$. Define a graded Lie algebra $L$ on 3 generators $y_{i}, i=0,1,2$, of degrees 1 , obeying 5 relations:

$$
\begin{aligned}
& {\left[y_{0}, y_{0}\right]=0, \quad\left[y_{0}, y_{1}\right]+\left[y_{1}, y_{0}\right]=0, \quad\left[y_{0}, y_{2}\right]+\left[y_{1}, y_{1}\right]+\left[y_{2}, y_{0}\right]=0,} \\
& {\left[y_{1}, y_{2}\right]+\left[y_{2}, y_{1}\right]=0, \quad\left[y_{2}, y_{2}\right]=0 .}
\end{aligned}
$$

It is concentrated in degrees 1 and 2: $L=L_{1} \oplus L_{2}$, where $L_{1}=V^{*}$ and $L_{2}=\mathbb{C} \cdot\left[y_{1}, y_{1}\right]$.

If $L^{\prime}=\mathbb{C} \cdot y_{1} \oplus \mathbb{C} \cdot\left[y_{1}, y_{1}\right] \subset L$ is a Lie subalgebra generated by $y_{1}$, then $L^{\prime}$ is free (sic!); it is an ideal, and the quotient algebra $\bar{L}=L / L^{\prime}$ is Abelian, on 2 generators $\bar{y}_{0}, \bar{y}_{2}$.

Consider the complex of the Chevalley cochains:

$$
C^{\cdot}(L)=\Lambda^{\cdot}\left(L^{*}\right)=S \cdot L_{1}^{*} \otimes \Lambda^{\cdot} L_{2}^{*}=S \cdot V \otimes \Lambda^{\cdot}\langle e\rangle, \quad e=\left[y_{1}, y_{1}\right]^{*}
$$

then one can identify $C^{\cdot}(L)$ with $K(B ; f)$.

Example 3.3. Starting from $b=2$ the product decomposition of the Hilbert series becomes infinite: for example, if $b=2$

$$
H\left(A_{2} ; t\right)=\frac{1+2 t}{(1-t)^{2}}=\frac{\left(1-t^{2}\right)^{3}\left(1-t^{4}\right)^{3}\left(1-t^{6}\right)^{11}\left(1-t^{8}\right)^{30}\left(1-t^{10}\right)^{105} \cdots}{(1-t)^{4}\left(1-t^{3}\right)^{2}\left(1-t^{5}\right)^{6}\left(1-t^{7}\right)^{18}\left(1-t^{9}\right)^{56} \ldots} .
$$

The first two exponents are "koszul": the number of unknowns and the number of equations. The exponents grow exponentially. 


\subsection{Gauss cyclotomic identity}

3.2.1. Necklaces. The necklace polynomial is defined as

$$
M_{n}(x)=\frac{1}{n} \sum_{d \mid n} \mu(d) x^{n / d} .
$$

Example 3.4. If $p$ is a prime, then $M_{p}(x)=\left(x^{p}-x\right) / p$.

Let a necklace $c$ be made of $n$ beads; suppose that each bead can be one of $m$ colors. A necklace is called primitive if it is not of the form $c=d c^{\prime}$ where $d \mid n, d>1$.

Theorem 3.5 (C. Moreau, 1872). The number of primitive necklaces made of $n$ beads in $b$ colors is equal to $M_{n}(b)$.

The proof is an exercise. C. Moreau was an artillery captain from Constantine, cf. [21].

Corollary 3.6. The number of all necklaces made of $n$ beads in $b$ colors is equal to $\Phi_{n}(b)$, where

$$
\Phi_{n}(x)=\frac{1}{n} \sum_{d \mid n} \phi(d) x^{n / d}
$$

$\phi(d)$ is the Euler function.

Proof. If this number is $C(n ; b)$ then

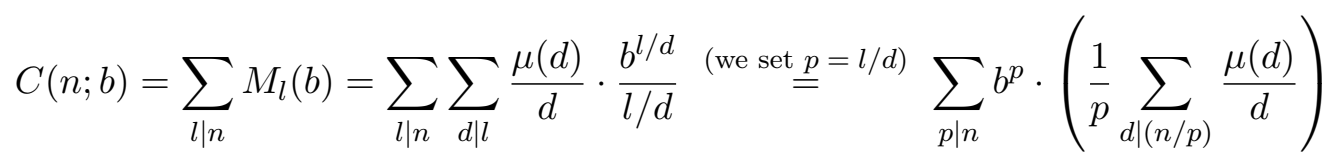

or,

$$
\sum_{d \mid(n / p)} \frac{\mu(d)}{d}=\frac{\phi(n / p)}{n / p}
$$

which proves the corollary.

3.2.2. A theorem of Pólya. Following Polyakov (cf. [28]), one can consider the same numbers from the point of view of Pólya theory [27].

Suppose we are given two finite sets $X$ and $Y$ as well as a weight function $w: Y \rightarrow \mathbb{N}$. If $n=|X|$, without loss of generality we can assume that $X=\{1,2, \ldots, n\}$. Consider the set of all mappings $F=\{f \mid f: X \rightarrow Y\}$. We can define the weight of a function $f \in F$ to be

$$
w(f)=\sum_{x \in X} w(f(x))
$$

Every subgroup of the symmetric group on $n$ elements, $S_{n}$, acts on $X$ through permutations. If $A$ is one such subgroup, an equivalence relation $\sim_{A}$ on $F$ is defined as $f \sim_{A} g \Longleftrightarrow f=g \circ a$ for some $a \in A$.

Denote by $[f]=\left\{g \in F \mid f \sim_{A} g\right\}$ the equivalence class of $f$ with respect to this equivalence relation. $[f]$ is also called the orbit of $f$. Since each $a \in A$ acts bijectively on $X$, then

$$
\left.w(g)=\sum_{x \in X} w(g(x))=\sum_{x \in X} w(g(a \circ x))\right)=\sum_{x \in X} w(f(x))=w(f) .
$$


Therefore we can safely define $w([f])=w(f)$. In other words, permuting the summands of a sum does not change the value of the sum.

Let $c_{k}=|\{y \in Y \mid w(y)=k\}|$ be the number of elements of $Y$ of weight $k$. The generating function by weight of the source objects is $c(t)=\sum_{k} c_{k} \cdot t^{k}$. Let $C_{k}=|\{[f] \mid w([f])=k\}|$ be the number of orbits of weight $k$. The generating function of the filled slot configurations is $C(t)=\sum_{k} C_{k} \cdot t^{k}$

Theorem 3.7. Given all the above definitions, Pólya's enumeration theorem asserts that

$$
C(t)=Z(A)\left(c(t), c\left(t^{2}\right), \ldots, c\left(t^{n}\right)\right)
$$

where $Z(A)$ is the cycle index (Zyklenzeiger) of $A$

$$
Z(A)\left(t_{1}, t_{2}, \ldots, t_{n}\right)=\frac{1}{|A|} \sum_{g \in A} t_{1}^{j_{1}(g)} t_{2}^{j_{2}(g)} \cdots t_{n}^{j_{n}(g)} .
$$

Consider the group of cyclic permutations $G \cong \mathbb{Z} / n$ as a subgroup of the symmetric group $S_{n}$. Define, after Pólya, the cycle index polynomial of $G$, in $n$ variables, as

$$
P_{G}\left(x_{1}, \ldots, x_{n}\right)=\frac{1}{n} \sum_{\sigma \in G} \prod_{i=1}^{n} x_{i}^{c_{i}(\sigma)}
$$

where $c_{i}(\sigma)$ is the number of cycles of length $i$ in $\sigma$.

In other words, one associates to each $\sigma \in G$ a monomial. For example, for $n=6$ there are following permutations:

$$
\begin{aligned}
& \sigma_{0}=(1)(2)(3)(4)(5)(6), \quad \text { the corresponding monomial is } x_{1}^{6}, \\
& \sigma_{1}=(123456): \quad x_{6}, \\
& \sigma_{2}=\sigma_{1}^{2}=(135)(246): \quad x_{3}^{2}, \\
& \left.\sigma_{3}=\sigma_{1}^{3}=(14)(25) 36\right): \quad x_{2}^{3} \\
& \sigma_{4}=\sigma_{1}^{4}=(153)(264): \quad x_{3}^{2} \\
& \sigma_{5}=\sigma_{1}^{5}=(654321): \quad x_{6} .
\end{aligned}
$$

The Zyklenzeiger is equal to $P_{\mathbb{Z} / 6}(x)=\frac{1}{6}\left(x_{1}^{6}+x_{2}^{3}+2 x_{3}^{2}+2 x_{6}\right)$.

In general,

$$
P_{\mathbb{Z} / n}\left(x_{1}, \ldots, x_{n}\right)=\frac{1}{n} \sum_{d \mid n} \phi(d) x_{d}^{n / d} .
$$

After a change of variables $x_{i}=\sum_{j=1}^{b} y_{j}^{i}$, one obtains a polynomial

$$
W_{G}\left(y_{1}, \ldots, y_{b}\right):=P_{G}\left(\sum y_{j}, \sum y_{j}^{2}, \ldots, \sum y_{j}^{n}\right)
$$

As a special case of the theorem of Pólya we obtain that the number of necklaces made of $n$ beads in $b$ colors is equal to $W_{\mathbb{Z} / n}(1, \ldots, 1)=P_{\mathbb{Z} / n}(b, \ldots, b)=\Phi_{n}(b)$. 


\subsubsection{Cyclotomic identity.}

Theorem 3.8 (Gauss, cf. [11]). One has the following formula:

$$
1-b t=\prod_{n=1}^{\infty}\left(1-t^{n}\right)^{M_{n}(b)} .
$$

It is proved by the application of the Möbius inversion. There is a useful generalization of this identity, found by Pieter Moree [22]. We will need it later studying the equivariant Hilbert series, see Section 3.4.4.

Theorem 3.9. Let $f(q) \in \mathbb{C}\left[q, q^{-1}\right]$ be an arbitrary Laurent polynomial. Introduce the polynomials

$$
M_{n}(f ; q)=\frac{1}{n} \sum_{d \mid n} \mu(d) f\left(q^{d}\right)^{n / d} .
$$

Then

$$
1-f(q) t=\prod_{n=1}^{\infty} \prod_{i=-\infty}^{\infty}\left(1-q^{i} t^{n}\right)^{a_{i n}}
$$

where for each $n$, the number $a_{i n}$ are defined by the equations

$$
\sum_{i} a_{i n} q^{i}=M_{n}(f ; q)
$$

Proof. Applying - $\log$ to the both sides of the above formula we obtain:

$$
-\log (1-f(q) t)=\sum_{m=1}^{\infty} \frac{f(q)^{m} t^{m}}{m}
$$

and

$$
-\log \left(\prod_{i, n}\left(1-q^{i} t^{n}\right)^{a_{i n}}\right)=\sum_{i} \sum_{n, k=1}^{\infty} a_{i n} \frac{q^{i k} t^{n k}}{k}=\sum_{m=1}^{\infty} t^{m} \cdot\left(\sum_{n \mid m} \sum_{i} a_{i n} \frac{q^{i m / n}}{m / n}\right),
$$

therefore

$$
f(q)^{m}=\sum_{n \mid m} \sum_{i} n a_{i n} q^{i m / n}
$$

for each $m=1,2, \ldots$. Make a change of variables: $p=q^{m}$,

$$
f\left(p^{1 / m}\right)^{m}=\sum_{n \mid m} \sum_{i} n a_{i n} p^{i / n} .
$$

Using the Möbius inversion we get:

$$
\sum_{i} n a_{i n} p^{i / n}=\sum_{d \mid n} \mu(n / d) f\left(p^{1 / d}\right)^{d}
$$

and making a substitution: $q=p^{1 / n}$, we obtain

$$
\sum_{i} n a_{i n} q^{i}=\sum_{d \mid n} \mu(n / d) f\left(q^{n / d}\right)^{d},
$$

which is the formula required. 
Moreover, O. Ogievetsky noticed that the theorem can be generalized to the case of several variables:

Theorem 3.10. Let $f\left(q_{1}, \ldots, q_{p}\right)$ be a Laurent polynomial in p variables. Introduce the Laurent polynomials

$$
M_{n}\left(f ; q_{1}, \ldots, q_{p}\right)=\frac{1}{n} \sum_{d \mid n} \mu(d) f\left(q_{1}^{d}, \ldots, q_{p}^{d}\right)^{n / d} .
$$

Then

$$
1-f\left(q, \ldots, q_{p}\right) t=\prod_{n=1}^{\infty} \prod_{i=-\infty}^{\infty}\left(1-q_{1}^{i_{1}} \cdots q_{p}^{i_{p}} t^{n}\right)^{a_{i_{1} \ldots i_{p} ; n}}
$$

where for each $n$, the numbers $a_{i_{1} \ldots i_{p} ; n}$ are the coefficients of $M_{n}$ :

$$
\sum_{i} a_{i_{1} \ldots i_{p} ; n} q_{1}^{i_{1}} \cdots q_{p}^{i_{p}}=M_{n}\left(f ; q_{1}, \ldots, q_{p}\right) .
$$

The proof is the same as before.

Example 3.11. Take $f(q)=-q$. Then

$$
1+q t=(1-q t)^{-1}\left(1-q^{2} t^{2}\right)
$$

where

$$
\frac{1}{n} \sum_{d \mid n}(-1)^{d} \mu(d)
$$

is equal to 0 if $n \geq 3,-1$ if $n=1$, and 1 if $n=2$.

3.2.4. Application: the $\zeta$ function of the affine line. Let $A:=\mathbb{F}_{p}[x]$; this ring is similar in many ways to $\mathbb{Z}$.

Nonzero ideals $I \subset A$ are in bijection with unitary polynomials $f(x), I=(f)$, and principal ideals correspond to irreducible ones. Set

$$
N(I):=\sharp(A / I)=p^{\operatorname{deg} f},
$$

and define

$$
\zeta(A ; s)=\sum_{I \subset A, I \neq 0} N(I)^{-s}=\sum_{f \text { unitary }} p^{-s \operatorname{deg} f} .
$$

There is $p^{n}$ unitary polynomials of degree $n$, therefore

$$
\zeta(A ; s)=\sum_{n=1}^{\infty} p^{n} \cdot p^{-s n}=\frac{1}{1-p \cdot p^{-s}}=\frac{1}{1-p T},
$$

where $T:=p^{-s}$.

The Euler product formula for $\zeta(A ; s)$ can be written in the following form

$$
\zeta(A ; s)=\prod_{f \text { unitary, irreducible }} \frac{1}{1-p^{-\operatorname{deg} f \cdot s}}
$$




$$
=\prod_{d=1}^{\infty} \prod_{f \text { un., irr., } \operatorname{deg} f=d} \frac{1}{1-p^{-d s}}=\prod_{d=1}^{\infty} \frac{1}{\left(1-T^{d}\right)^{N_{d}(p)}}
$$

where $N_{d}(p)$ denotes the number of unitary irreducible polynomials of degree $d$ in $A$.

On the other hand, applying the cyclotomic identity to (3.4), one gets

$$
\zeta(A ; s)=\frac{1}{1-p T}=\frac{1}{\prod_{d=1}^{\infty}\left(1-T^{d}\right)^{M_{d}(p)}},
$$

proving therefore

Theorem 3.12 (Gauss [11]). The number $N_{d}(p)$ of irreducible unitary polynomials of degree $d$ in $\mathbb{F}_{p}[x]$ is equal to

$$
M_{d}(p)=\frac{1}{d} \sum_{l \mid d} \mu(l) p^{d / l} .
$$

Corollary 3.13. For $d \geq 1, N_{d}(p)>0$, i.e. for each $d \geq 1$ there is an irreducible polynomial of degree $d$.

It is interesting to compare this theorem of Gauss with Riemann's explicit formula:

$$
\pi(x)=\sum_{n=1}^{\infty} \frac{\mu(n)}{n}\left\{\operatorname{Li}\left(x^{1 / n}\right)+\sum_{\mathfrak{I m}(\rho)>0}\left(x^{\rho / n}+x^{1-\rho}\right)+\int_{x^{1 / n}}^{\infty} \frac{d t}{t\left(t^{2}-1\right) \log t}-\log 2\right\} .
$$

Here $\pi(x)=$ the number of primes $p \leq x$, the summation is over the non-trivial roots of $\zeta(s)$, and

$$
\operatorname{Li} x=\int_{1}^{x} \frac{d t}{\log t} .
$$

\subsection{Witt formula}

La Nature est un temple où de vivants piliers Laissent parfois sortir de confuses paroles...

Ch. Baudelaire

Theorem 3.14 (Witt [35]). Let $L$ be a free Lie algebra on $b$ generators. Then

$$
\operatorname{dim} L_{n}=M_{n}(b)=\frac{1}{n} \sum_{d \mid n} \mu(d) b^{n / d} .
$$

In other words,

$$
H(L ; t):=\sum_{n=0}^{\infty} \operatorname{dim} L_{n} \cdot t^{n}=-\sum_{m=1}^{\infty} \frac{\mu(m)}{m} \log \left(1-b t^{m}\right) .
$$

Proof. If $x_{1}, \ldots, x_{b}$ are the generators of $L$, then the universal enveloping algebra $U L=$ $\mathbb{C}\left\langle x_{1}, \ldots, x_{b}\right\rangle$ (a free associative algebra), has the following Hilbert series

$$
H(U L ; t):=\sum_{i=0}^{\infty} \operatorname{dim} U L_{i} \cdot t^{i}=\frac{1}{1-b t} .
$$


On the other hand, due to Poincaré-Birkhoff-Witt, $U L=S L$, therefore, if one denotes $a_{n}:=$ $\operatorname{dim} L_{n}$, then

$$
H(U L ; t)=\prod_{n=1}^{\infty} \frac{1}{\left(1-t^{n}\right)^{a_{n}}},
$$

and applying the cyclotomic identity finishes the proof.

3.3.1. This theorem admits a nice generalization. Let $L$ be a free Lie superalgebra with $b$ even generators $x_{1}, \ldots, x_{b}$ and $c$ odd generators $y_{1}, \ldots, y_{c}$.

Equip $L$ with a grading, assigning to $x_{i}$ and to $y_{j}$ the degree 1 , and setting $\operatorname{deg}([a, b])=$ $\operatorname{deg}(a)+\operatorname{deg}(b)$. If $L_{n} \subset L$ is a subspace of the elements of the degree $n$, then $L=\bigoplus_{n=1}^{\infty} L_{n}$.

Explicitly, the Lie monomial $\alpha=\left[a_{1},\left[a_{2}, \ldots a_{n}\right] \ldots\right]$, where $a_{i}=x_{j}$ or $y_{k}$, has the degree $n$. On the other hand, its parity is equal to the number of $y$ 's among $a_{i}$. For example, $\left[x_{i}, y_{j}\right]$ is an element odd of degree 2 (sic!).

Each homogenous summand $L_{n}$ therefore is decomposed into two summands: even and odd elements, $L_{n}=L_{n}^{p} \oplus L_{n}^{i}$. One defines the super-dimension as $\operatorname{dim}^{\sim} L_{n}=\operatorname{dim} L_{n}^{p}-\operatorname{dim} L_{n}^{i}$.

Theorem 3.15 (Petrogradsky [25]). One has the formula

$$
\operatorname{dim}^{\sim} L_{n}=M_{n}(b-c)=\frac{1}{n} \sum_{d \mid n} \mu(d)(b-c)^{n / d} .
$$

Example 3.16. Let $b=0, c=1$; then $L=\mathbb{C} \cdot y \oplus \mathbb{C} \cdot[y, y]$. In the right hand side, one sees that $M_{n}(-1)=-1$ if $n=1,1$ if $n=2$, and 0 if $n \geq 3$, cf. Examples 3.11 and 3.2.

3.3.2. Hochschild homology. Let $A$ be an associative unitary algebra. Recall that the Hochschild homology $H H_{i}(A)$ of $A$ is defined as the homology of the complex

$$
C H .(A): \cdots \longrightarrow A \otimes A \otimes A \stackrel{b}{\longrightarrow} A \otimes A \stackrel{b}{\longrightarrow} A \longrightarrow 0,
$$

where $C H_{n}(A)=A^{\otimes n+1}$, and the differential $b$ is defined by

$$
\begin{gathered}
b\left(a_{1} \otimes a_{2} \cdots \otimes a_{n}\right)=a_{1} a_{2} \otimes a_{3} \otimes \cdots \otimes a_{n}-a_{1} \otimes a_{2} a_{3} \otimes a_{4} \cdots \otimes a_{n}+\cdots \\
+(-1)^{n-1} a_{1} \otimes \cdots \otimes a_{n-1} a_{n}+(-1)^{n} a_{n} a_{1} \otimes a_{2} \otimes \cdots \otimes a_{n-1} .
\end{gathered}
$$

In particular, $d(a \otimes b)=a b-b a$ and $H H_{0}(A)=A /[A, A]$, where $[A, A] \subset A$ is the subspace generated by the elements $a b-b a$.

In fact, one can replace $A$ by $\bar{A}:=A / \mathbb{C}$ : the complex $C H .(A)$ is quasi-isomorphic to

$$
\overline{C H} .(A): \cdots \longrightarrow A \otimes \bar{A} \otimes \bar{A} \longrightarrow \bar{A} \otimes A \longrightarrow A \longrightarrow 0 .
$$

One has

$$
H H_{i}(A)=\operatorname{Tor}_{i}^{A \otimes A^{o}}(A, A) .
$$

Let us introduce the operators $B: A^{\otimes n+1} \longrightarrow A^{\otimes n+2}$ by the formula:

$$
\begin{aligned}
B\left(a_{0}, \ldots, a_{n}\right)= & \sum_{i=0}^{n-1}\left\{(-1)^{n i}\left(1, a_{i}, \ldots, a_{n}, a_{0}, \ldots, a_{i-1}\right)\right. \\
& \left.-(-1)^{n(i-1)}\left(a_{i-1}, 1, a_{i}, \ldots, a_{n}, a_{0}, \ldots, a_{i-2}\right)\right\} .
\end{aligned}
$$


For example,

$$
B\left(a_{0}\right)=\left(1, a_{0}\right)+\left(a_{0}, 1\right) .
$$

Then $B b+b B=0$ (cf. [17, 2.1]); therefore it induces the morphisms

$$
B: H H_{n}(A) \longrightarrow H H_{n+1}(A) \text {. }
$$

Recall that the cyclic homology can be defined as the homology of the bi-complex

$$
H C_{i}(A)=H_{i}(C H .(A) \stackrel{B}{\longrightarrow} C H .(A)[1] \stackrel{B}{\longrightarrow} C H .(A)[2] \stackrel{B}{\longrightarrow} \cdots) .
$$

3.3.3. Now one can give an algebraic interpretation of the polynomials $\Phi_{n}(x)=\sum_{d \mid n} \phi(d) x^{n / d} / n$.

Let $A=\mathbb{C}\left\langle x_{1}, \ldots, x_{b}\right\rangle$, then $H H_{0}(A)$ inherits a grading from $A, H H_{0}(A)=\bigoplus_{n=0}^{\infty} H H_{0}(A)_{n}$; if one has $\bar{A}=\underset{n \geq 1}{\oplus} A_{n}$, then:

$$
\overline{H H_{0}}(A)=\underset{n \geq 1}{\oplus} H H_{0}(A)_{n}=\bar{A} /[\bar{A}, \bar{A}] .
$$

One can think of $H H_{0}(A)_{n}$ as a space of cyclic words $x_{i_{1}} x_{i_{2}} \ldots x_{i_{n}}$ of length $n$ in letters $x_{i}$, where two words are identified if one is a cyclic permutation of another. Therefore cyclic words are identified with necklaces of $n$ beads in $b$ colors.

Hence $H H_{0}(A)_{n}$ can be viewed as a linear space with a basis indexed by necklaces of $n$ beads in $b$ colors. It follows that

$$
\operatorname{dim} H H_{0}(A)_{n}=\Phi_{n}(b)=\frac{1}{n} \sum_{d \mid n} \phi(d) b^{n / d}, \quad n \geq 1,
$$

(cf. Corollary 3.6), or

$$
H\left(\bar{H} H_{0}(A) ; t\right)=-\sum_{m=1}^{\infty} \frac{\phi(m)}{m} \log \left(1-b t^{m}\right)
$$

(the Polyakov formula).

3.3.4. Let $V=\bigoplus_{i=1}^{b} \mathbb{C} \cdot x_{i}$, therefore $A=T V$. For each $n \geq 1$ one defines an automorphism $\tau: V^{\otimes n} \longrightarrow V^{\otimes n}$ as

$$
\tau\left(v_{1} \otimes \cdots \otimes v_{n}\right)=v_{n} \otimes v_{1} \otimes v_{2} \otimes \cdots \otimes v_{n-1} .
$$

One observes that

$$
H H_{0}(T V)_{n}=V_{\tau}^{\otimes n}:=\operatorname{Coker}(1-\tau) .
$$

Following [17, 3.1], one defines a complex of length 1:

$$
C H^{s m}(T V): 0 \longrightarrow T V \otimes V \longrightarrow T V \longrightarrow 0
$$

equipped with a differential $d(a \otimes v)=a v-v a$. Define also a morphism of complexes $\phi$ : $C H .(T V) \longrightarrow C H^{s m}(T V)$ as: $\phi_{0}=\operatorname{Id}_{T V}$,

$$
\phi_{1}\left(a \otimes v_{1} \ldots v_{n}\right)=\sum_{i=1}^{n} v_{i+1} \cdots v_{n} a v_{1} \cdots v_{i-1} \otimes v_{i} .
$$


On the other hand, there is an evident inclusion $\iota: C H^{s m}(T V) \longrightarrow C H .(A)$ such that $\phi \circ \iota=\mathrm{Id}$, and, as one can check, $\iota \circ \phi$ is homotopic to the identity.

It follows that

$$
H H_{1}(T V)_{n}=\left(V^{\otimes n}\right)^{\tau}:=\operatorname{Ker}(1-\tau)
$$

and $H H_{i}(T V)=0$ for $i \geq 2$.

Instead of the Hochschild homology one can also consider the (reduced) cyclic homology. Then: $H C_{0}(T V)=H H_{0}(T V)$ and $H_{i}(T V)=0$ for $i>0$, cf. [23].

3.3.5. Partial derivatives. Let $m=\ldots x_{i} x_{j} x_{k} \ldots$ be a cyclic word (i.e. $m \in T V /[T V, T V]$ ) such that the letter $x_{i}$ appears once in it; then one can define a usual word (i.e. an element de $T V$ ) $\partial m / \partial x_{i}$, by "cutting" $m$ and removing the letter $x_{i}$ :

$$
\partial m / \partial x_{i}=x_{j} x_{k} \ldots
$$

When $x_{i}$ appears in a word several times, the result will be a sum, by the Leibnitz rule, cf. [19]. In this way we define a map

$$
\frac{\partial}{\partial x_{i}}: T V /[T V, T V] \longrightarrow T V .
$$

Consider an operator $\sum_{i=1}^{b} x_{i} \partial / \partial x_{i}$ (cf. (3.8)). It respects the polynomial degree, and it is not hard to verify that its image is contained in $T V^{\tau}$. For example:

$$
e(x y z)=x y z+y z x+z x y .
$$

One observes that the map

$$
\sum_{i=1}^{b} x_{i} \frac{\partial}{\partial x_{i}}: H H_{0}(T V)=T V_{\tau} \longrightarrow T V^{\tau}=H H_{1}(T V)
$$

coincides with homomorphism B, cf. (3.6), (3.7). (Compare [23, the line before (14), p. 8].)

It was pointed out to us by V. Ginzburg that there is another interpretation of the above map. Consider the composition

$$
d: T V_{\tau}=T V /[T V, T V] \stackrel{\sum x_{i} \partial / \partial x_{i}}{\longrightarrow} T V^{\tau} \subset T V \otimes V
$$

It was already Quillen who studied this map in the 80's, cf. [30]. In the notations of [30, $\S 3]$ $T V /[T V, T V]=T V_{\natural}$ and $T V \otimes V=\Omega_{T V, \mathfrak{t}}^{1}$; one can consider these spaces as the space of cyclic functions (differentiable 1-forms respectively) over the "non-commutative space Spec $T V$ "; the morphism (3.10) is called "the Karoubi-de Rham differential".

\subsection{Koszul duality}

3.4.1. Let $A$ be an associative quadratic algebra, which means that, it is a quotient $A=$ $T V /(R)$ of the free associative algebra over the space $V$ of finite dimension by the two-sided ideal $(R)$ generated by a subspace of relations $R \subset V \otimes V$.

Recall that the quadratic dual algebra is defined as $A^{!}=T V^{*} /\left(R^{\perp}\right)$, where $R^{\perp} \subset V^{*} \otimes V^{*} \cong$ $(V \otimes V)^{*}$ is the annihilator of $R$.

We are interested in the case when $A$ is commutative; in this case $R \supset \Lambda^{2} V$, and $R^{\perp} \subset$ $\Lambda^{2} V^{\perp}=S^{2} V^{*}$. In other words, if $\left\{x_{i}\right\}$ form a basis of $V,\left\{y_{i}\right\}$ form a basis of $V^{*}$, then $R^{\perp}$ 
is contained in $S^{2} V^{*} \subset V^{\otimes 2}$; in other words it is generated by odd commutators $\left[y_{i}, y_{j}\right]=$ $y_{i} y_{j}+y_{i} y_{j}$. Define a Lie algebra $L$ as a graded Lie algebra generated by $y_{i}$ of degree 1 and relations $g=0, g \in R^{\perp}$. Then $A^{!}=U L$ by definition. The Lie algebra $L$ is called the Koszul, or Quillen dual of $A$.

For example, if $A=H^{*}(X ; \mathbb{C})$ is the cohomology ring of a simply connected topological space $X$, then $L$ is its homotopy Lie algebra, $\oplus \pi_{i}(X)_{\mathbb{C}}$, under some additional conditions, cf. [29].

Therefore we have, $L=\bigoplus_{n=1}^{\infty} L_{n}$; moreover one observes that $L_{1}=V^{*}, L_{2}=S^{2} V^{*} / R^{\perp}$. Set $L_{\leq 2}=L_{1} \oplus L_{2}$; it is a quotient Lie algebra of $L$.

3.4.2. The Chevalley cochain complex of $L$ is by definition the space

$$
C^{\cdot}(L)=S L_{1}^{*} \otimes \Lambda L_{2}^{*} \otimes S L_{3}^{*} \otimes \cdots
$$

equipped with the Chevalley differential. This complex is double graded:

$$
C \cdot(L)=\underset{a, b=0}{\oplus} C^{\cdot}(L)_{a b}
$$

where the first (homological) degree of $L_{i}^{*}$ is set to be equal $i-1$, and the second (polynomial) degree of $L_{i}^{*}$ is $i$, both gradings are compatible with the product. The Chevalley differential preserves the second grading and decreases the first by 1 :

$$
d C \cdot(L)_{a b} \subset C^{\cdot}(L)_{a-1, b} .
$$

Here are the components of $C \cdot(L)$ of polynomial degree $\leq 3$ :

$$
\begin{aligned}
& L_{3}^{*} \longrightarrow L_{2}^{*} \otimes L_{1}^{*} \longrightarrow S^{3} L_{1}^{*}, \\
& L_{2}^{*} \longrightarrow S^{2} L_{1}^{*}, \\
& L_{1}^{*} .
\end{aligned}
$$

One has: $C^{\cdot}(L)_{0}=S L_{1}^{*}$ and the complex starts as:

$$
\cdots \longrightarrow\left(L_{3}^{*} \oplus \Lambda^{2} L_{2}^{*}\right) \otimes S L_{1}^{*} \longrightarrow L_{2}^{*} \otimes S L_{1}^{*} \longrightarrow S L_{1}^{*} .
$$

Since $L_{1}^{*}=V$ and $L_{2}^{*}=R \subset S^{2} V$, the first differential in (3.12) is a $S V$-linear map which sends $f \otimes 1 \in L_{2}^{*}$ to $f \in R \subset S^{2} L_{1}^{*}$.

One observes that:

(a) there is a natural augmentation $C^{\cdot}(L) \longrightarrow A=S V /(R)$, cf. [24].

(A similar picture arises in the construction of the mixed Tate motivic cohomology of a field $k$, cf. [4]. There, the analogues of complexes $C \cdot(L)_{n}$. are the Beilinson motivic complexes $\mathbb{Z}(n)$, the analogue of $A$ is the Milnor $K$-theory $K^{\mathrm{Miln}}(k)$; the analogue of $L$ is (the Lie algebra of) the "mixted Tate motivic fundamental group".)

(b) If one chooses a basis $\left\{f_{1}, \ldots, f_{d}\right\}$ of $R$, then one can identify the Chevalley complex of $L_{\leq 2}$ with the Koszul complex

$$
C \cdot\left(L_{\leq 2}\right) \stackrel{\simeq}{=}(S V ;(f)),
$$

cf. Example 3.2.

(c) There is a natural inclusion $C^{\cdot}\left(L_{\leq 2}\right) \subset C^{\cdot}(L)$ compatible with the augmentation to $A$.

Define a generating series

$$
H(C \cdot(L) ; u, t):=\sum_{a, b=0}^{\infty} \operatorname{dim} C \cdot(L)_{a b} u^{a} t^{b}
$$


and its specialization, the Euler-Hilbert series:

$$
E H\left(C^{\cdot}(L) ; t\right):=H\left(C^{\cdot}(L) ;-1, t\right)=\sum_{n=0}^{\infty} E P\left(C^{\cdot}(L)_{?, n}\right) t^{n},
$$

where EP stands for the Euler-Poincaré characteristic. One observes immediately that

$$
E H\left(S L_{n}^{*}\right)=\left(1-t^{n}\right)^{-\operatorname{dim} L_{n}} \quad \text { and } \quad E H\left(\Lambda L_{n}^{*}\right)=\left(1-t^{n}\right)^{\operatorname{dim} L_{n}},
$$

where

$$
E H(C \cdot(L) ; t)=\prod_{n=1}^{\infty}\left(1-t^{n}\right)^{(-1)^{n} \operatorname{dim} L_{n}} .
$$

3.4.3. For example, consider the Veronese ring $A_{b}$, cf. Section 3.1.1. Recall that $A_{b}$ is generated by $x_{0}, \ldots, x_{b+1}$, subject to relations $x_{i} x_{j}-x_{k} x_{l}=0$ if $i+j=k+l$. Then $A^{!}$is generated by $y_{i}=x_{i}^{*}, 0 \leq i \leq b+1$ obeying the relations

$$
\sum_{i+j=k}\left[y_{i}, y_{j}\right]=0, \quad k=0, \ldots, 2(b+1),
$$

where $\left[y_{i}, y_{j}\right]=y_{i} y_{j}+y_{j} y_{i}\left(\right.$ sic!). Therefore if one defines a graded Lie algebra $L^{b}$ with generators $y_{i} \in L_{1}^{b}=A_{b 1}^{*}$ and relations (3.14), then $A_{b}^{!}=U L^{b}$.

This Lie algebra has a nice structure, cf. [13] (the case $b=1$ was considered in Example 3.2). It admits an involution $i\left(y_{j}\right)=y_{b+1-j}$. Let $\hat{L}^{b} \subset L^{b}$ be a Lie subalgebra generated by $y_{1}, \ldots, y_{b}$. Then $\hat{L}^{b}=\left[L^{b}, L^{b}\right]$ is a Lie ideal (stable under $i$ ); as a graded Lie algebra it is free.

The quotient algebra $\bar{L}=L^{b} / \hat{L}^{b}$ is a graded Abelian Lie algebra on 2 generators $\bar{y}_{0}, \bar{y}_{b+1}$. One sees that $\hat{L}_{\geq 2}^{b}=L_{\geq 2}^{b}$.

We have noticed in Example 3.2 that $L_{1}$ is finite-dimensional; as opposed to that, if $b \geq 2$, than $L^{b}$ is infinite-dimensional (with an exponential growth). Namely, one has $L^{b}=\underset{n=1}{\oplus} L_{b n}$, where the dimensions of the homogenous components can be calculated easily using an odd analogue of the Witt theorem, see Theorem 3.14. One obtains:

$$
\begin{aligned}
& \operatorname{dim} L_{1}=-M_{1}(-b)+2=b+2, \\
& \operatorname{dim} \sim L_{n}=\operatorname{dim}^{\sim} \hat{L}_{n}=M_{n}(-b)=\frac{1}{n} \sum_{d \mid n} \mu(d)(-b)^{n / d} \quad(n \geq 2) .
\end{aligned}
$$

Here $\hat{L}_{b 1}$ is generated by $b$ odd elements, therefore

$$
\operatorname{dim} \sim \hat{L}_{n}=(-1)^{n} \operatorname{dim} \hat{L}_{n} .
$$

One observes that the signs od $\operatorname{dim}^{\sim} L_{n}$ alternate. For example, $\operatorname{dim} L_{2}=M_{2}(-b)=\left(b^{2}+b\right) / 2$, $\operatorname{dim} L_{3}=-M_{3}(-b)=\left(b^{3}-b\right) / 3$, etc.

Now consider the Chevalley complex of $L^{b}:(3.13)$ implies that its Euler-Hilbert is as follows:

$$
\begin{gathered}
E H\left(C \cdot\left(L^{b}\right) ; t\right)=(1-t)^{-\operatorname{dim} L_{b 1}}\left(1-t^{2}\right)^{\operatorname{dim} L_{b 2}}\left(1-t^{3}\right)^{-\operatorname{dim} L_{b 3}} \ldots \\
=(1-t)^{-2} \prod_{n=1}^{\infty}\left(1-t^{n}\right)^{M_{n}(-b)}=\frac{1+b t}{(1-t)^{2}} .
\end{gathered}
$$

It is clear that it coincides with the Hilbert series of the ring $A_{b},(3.1)$. the last equality is due to the cyclotomic identity. It is not surprising.

In fact, a deep theorem of Bezrukavnikov says: 
Theorem 3.17 (cf. [6]). The algebra $A_{b}$ is Koszul.

Formula (3.15) can be viewed as a "numerical evidence" that the theorem holds.

Corollary 3.18. There are natural isomorphisms $A_{b}^{!} \cong \operatorname{Ext}_{A_{b}}(\mathbb{C}, \mathbb{C}), A_{b} \cong \operatorname{Ext}_{A_{b}^{!}}(\mathbb{C}, \mathbb{C})=$ $H^{\cdot}(L ; \mathbb{C})$.

It follows that $A_{b}^{\sim}:=C^{\cdot}\left(L^{b}\right)$ is a dga resolution of $A_{b}$, cf. [24].

3.4.4. Characters. The Lie algebra $\mathrm{sl}(2)$ acts on $A_{b}$ in such a way that $A_{b 1}$ is an irreducible sl(2)-module; therefore its character is

$$
\operatorname{Ch}\left(A_{b 1}\right)=[b+1]_{q}=\frac{q^{b+1}-q^{-b-1}}{q-q^{-1}} .
$$

The character of $A_{b}$ is given by the equivariant Hilbert series, cf. (3.2):

$$
H_{q}\left(A_{b} ; t\right)=\frac{1+[b]_{q} t}{\left(1-q^{b+1} t\right)\left(1-q^{-b-1} t\right)} .
$$

The above action induces an action of $\operatorname{sl}(2)$ on $L^{b}$ and therefore on $A_{b}^{\sim}:=C^{\cdot}\left(L^{b}\right)$. Applying now the " $q$-cyclotomic" identity: Theorem 3.9 for $f(q)=-[b]_{q}$, one obtains the $\operatorname{sl}(2)$-character $\mathrm{Ch}\left(A_{b}^{\sim}\right)$.

On the other hand:the subalgebra Lie $\hat{L}_{b} \subset L^{b}$ is free on $b$ generators, therefore the Lie algebra $\mathrm{gl}(b)$ acts on it. One can use the theorem of Ogievetsky (see Theorem 3.10) for $f\left(q_{1}, \ldots, q_{b}\right)=$ $-\mathrm{Ch}_{\mathrm{gl}(b)}\left(\hat{L}_{b 1}\right)$ for calculation of the character $\mathrm{Ch}_{\mathrm{gl}(b)}\left(C^{\cdot}\left(\hat{L}_{b}\right)\right)$.

The $\mathrm{gl}(b)$-character of the free Lie algebra on odd $b$ generators was calculated by Angeline Brandt in [9].

The free group on $b$ generators is isomorphic to the fundamental group of the Riemann sphere with $b+1$ points removed; its nilpotent completion is a fundamental object of the theory of Grothendieck-Drinfeld-Ihara, cf. [10].

3.4.5. Returning to the case of an arbitrary commutative quadratic algebra, one can show that a complete intersection is Koszul, cf. [26]. The other way around, if $A$ is commutative Koszul, and $L$ is its dual Lie algebra, then $A$ is a complete intersection if and only if $L=L_{\leq 2}$.

One can say that commutative (or maybe also noncommutative?) Koszul algebras are natural generalizations of the quadratic complete intersections; and one would expect that all the results which hold for quadratic complete intersections will generalize to Koszul algebras.

\section{Acknowledgements}

We thank Fedor Malikov who read thoroughly the first part and helped to correct many signs; some calculations made with him have been the starting point of the second part. We are grateful to Vladimir Hinich for very interesting discussions about Golod rings and Koszul algebras; to Oleg Ogievetsky for an important remark; to Alexander Polishchuk for very useful consultations; to Hossein Abbaspour and Thomas Tradler who taught us about the string topology, and especially to Victor Ginzburg for his numerous explanations, questions and bibliographical comments.

This article was finished during our stay at Max-Planck-Institut für Mathematik and the Hausdorff Institut für Mathematik in June and July 2008; we are grateful to both institutions for the excellent working atmosphere. 


\section{References}

[1] Aisaka Yu., Arroyo E.A., Berkovits N., Nekrasov N., Pure spinor partition function and the massive superstring spectrum, J. High Energy Phys. 2008 (2008), no. 8, 050, 72 pages, arXiv:0806.0584.

[2] Beauville A., Laszlo Y., Conformal blocks and generalized theta functions, Comm. Math. Phys. 164 (1994), 385-419, alg-geom/9309003.

[3] Beilinson A., Drinfeld V., Chiral algebras, American Mathematical Society Colloquium Publications, Vol. 51, American Mathematical Society, Providence, RI, 2004.

[4] Beilinson A.A., Goncharov A.B., Schechtman V.V., Varchenko A.N., Aomoto dilogarithmes, mixed Hodge structures, and motivic cohomology of pairs of triangles on the plane, in The Grothendieck Festschrift, Vol. I, Progr. Math., Vol. 86, Birkhäuser Boston, Boston, MA, 1990, 135-172.

[5] Berkovits N., Nekrasov N.A., The character of pure spinors, Lett. Math. Phys. 74 (2005), 75-109, hep-th/0503075.

[6] Bezrukavnikov R., Koszul property and Frobenius splitting of Schubert varieties, alg-geom/9502021.

[7] Cohen R.L., Jones J.D.S., A homotopy theoretic realisation of string topology, Math. Ann. 324 (2002), 773-798, math.GT/0107187.

[8] Chas M., Sullivan D., String topology, math.GT/9911159.

[9] Brandt A.J., The free Lie ring and Lie representations of the full linear group, Trans. Amer. Math. Soc. 56 (1944), 528-536.

[10] Drinfeld V.G., On quasitriangular quasi-Hopf algebras and on a group that is closely connected with $\operatorname{Gal}(\overline{\mathbb{Q}} / \mathbb{Q})$, Algebra i Analiz 2 (1990), no. 4, 149-181 (English transl.: Leningrad Math. J. 2 (1991), no. 4, 829-860).

[11] Gauss C.F., Disquisitiones generales de congruentis, Analysis residuorum. Caput octavum, Collected Works, Vol. 2, Georg Olms Verlag, Hildersheim - New York, 1973, 212-242.

[12] Golyshev V., The canonical strip. I, arXiv:0903.2076.

[13] Gorodentsev A.L., Khoroshkin A.S., Rudakov A.N., On syzygies of highest weight orbits, Amer. Math. Soc. Transl. Ser. 2, Vol. 221, Amer. Math. Soc., Providence, RI, 2007, 79-120, math.AG/0602316.

[14] Hardy G.H., Divergent series, The Clarendon Press, Oxford, 1949.

[15] Hirzebruch F., Neue topologische Methoden in der algebraischen Geometrie, Ergebnisse der Mathematik und ihrer Grenzgebiete (N.F.), Heft 9, Springer-Verlag, Berlin - Göttingen - Heidelberg, 1956.

[16] Hirzebruch F., Private communication, 2008.

[17] Loday J.-L., Cyclic homology, Springer-Verlag, Berlin, 1992.

[18] Kaufmann R.M., A proof of a cyclic version of Deligne's conjecture via cacti, math.QA/0403340.

[19] Kontsevich M., Formal (non)commutative symplectic geometry, The Gelfand Mathematical Seminars, 19901992, Editors L. Corwin et al., Birkhäuser Boston, Boston, MA, 1993, 173-188.

[20] Lian B.H., Zuckerman G.J., New perspectives on the BRST-algebraic structure of string theory, Comm. Math. Phys. 154 (1993), 613-646, hep-th/9211072.

[21] Moreau C., Sur les permutations circulaires distinctes, Nouv. Ann. Math. 11 (1872), 309-314.

[22] Moree P., On the average number of elements in a finite field with order or index in a prescribed residue class, Finite Fields Appl. 10 (2004), 438-463, math.NT/0212220.

[23] Movshev M., On deformations of Yang-Mills algebras, hep-th/0509119.

[24] Movshev M., Schwarz A., Algebraic structure of Yang-Mills theory, hep-th/0404183.

[25] Petrogradsky V.M., On Witt's formula and invariants for free Lie superalgebras, in Formal Power Series and Algebraic Combinatorics (Moscow, 2000), Springer, Berlin, 2000, 543-551.

[26] Polishchuk A., Positselski L., Quadratic algebras, University Lecture Series, Vol. 37, American Mathematical Society, Providence, RI, 2005.

[27] Pólya G., Kombinatorische Anzahlbestimmungen für Gruppen, Graphen und chemische Verbindungen, Acta Math. 68 (1937), 145-254.

[28] Polyakov A., Gauge fields and space-time, Internat. J. Modern Phys. 17 (2002), suppl., 119-136, hep-th/0110196.

[29] Quillen D., Rational homotopy theory, Ann. Math. (2) 90 (1969), 205-295. 
[30] Quillen D., Algebra cochains and cyclic cohomology, Inst. Hautes Études Sci. Publ. Math. (1988), no. 68, 139-174.

[31] Ramis J.-P., Séries divergentes et théories asymptotiques, Bull. Soc. Math. France 121 (1993), suppl., 74 pages.

[32] Tradler T., Zeinallian M., On the cyclic Deligne conjecture, J. Pure Appl. Algebra 204 (2006), 280-299, math.QA/0404218.

[33] Weil A., Elliptic functions according to Eisenstein and Kronecker, Ergebnisse der Mathematik und ihrer Grenzgebiete, Vol. 88, Springer-Verlag, Berlin - New York, 1976.

[34] Whittaker E.T., Watson G.N., A course of modern analysis. An introduction to the general theory of infinite processes and of analytic functions; with an account of the principal transcendental functions, reprint of 4th ed. (1927), Cambridge Mathematical Library, Cambridge University Press, Cambridge, 1996.

[35] Witt E., Treue Darstellung Liescher Ringe, J. Reine Angew. Math. 177 (1937), 152-160.

[36] Zagier D., Elementary aspects of the Verlinde formula and of the Harder-Narasimhan-Atiyah-Bott formula, in Proceedings of the Hirzebruch 65 Conference on Algebraic Geometry (Ramat Gan, 1993), Israel Math. Conf. Proc., Vol. 9, Bar-Ilan Univ., Ramat Gan, 1996, 445-462. 